\title{
A class of Neumann type systems and its application
}

\author{
Jinbing Chen
}

Communicated by Tudor Ratiu, received December 18, 2011.

\begin{abstract}
A class of Neumann type systems are derived separating the spatial and temporal variables for the $2+1$ dimensional Caudrey-Dodd-GibbonKotera-Sawada (CDGKS) equation and the modified Korteweg-de Vries (mKdV) hierarchy. The Lax-Moser matrix of Neumann type systems is worked out, which generates a sequence of integrals of motion and a hyperelliptic curve of KdV type. We deduce the constrained Hamiltonians to put Neumann type systems into canonical Hamiltonian equations and further complete the Liouville integrability for the Neumann type systems. We also specify the relationship between Neumann type systems and infinite dimensional integrable systems (IDISs), where the involutivity solutions of Neumann type systems yield the finite parametric solutions of IDISs. From the Abel-Jacobi variables, the evolution behavior of Neumann type flows are shown on the Jacobian of a Riemann surface. Finally, the Neumann type flows are applied to produce some explicit solutions expressed by Riemann theta functions for the $2+1$ dimensional CDGKS equation and the mKdV hierarchy.
\end{abstract}

\section{Contents}

1. Introduction

2. The Lax representations of INLEEs 149

3. The Neumann type systems 152

4. The Liouville integrability 155

5. Relation between Neumann type systems and IDISs 160

6. The application of Neumann type flows 162

7. Conclusions and Discussions 167

Appendix A. An alternative proof of the proposition 2 168

$\begin{array}{ll}\text { Acknowledgements } & 169\end{array}$

$\begin{array}{ll}\text { References } & 169\end{array}$

1991 Mathematics Subject Classification. 35Q51, 37K10, 37K20.

Key words and phrases. Neumann type system, Dirac-Poisson bracket, Liouville integrability, Abel-Jacobi variable, explicit solution. 


\section{Introduction}

The Neumann system on $S^{2}$ is a mechanical system discovered in the 19th century [1]. From the McKean-Trubowitz identity given by the left-hand side endpoints of spectral gaps [2], in 1978 Moser generalized the Neumann system of 2 degrees of freedom to the arbitrary $N$ case [3], where the used Neumann map was

$$
u=\langle q, q\rangle-\langle\Lambda p, p\rangle,
$$

which constitutes the finite-gap potential of the KdV equation [4]. Based on the relationship between the KdV equation and the Neumann system, Cao normalized the Neumann map (1.1) into the form (here $g_{-1}$ is the first element of Lenard gradients, and $\nabla \lambda_{j}$ is the functional gradient of eigenvalue) [5]

$$
g_{-1}=\sum_{j=1}^{N} \nabla \lambda_{j}
$$

to get the finite dimensional integrable system (FDIS) of Neumann type attached with a geometry condition likes that of the classical Neumann system, in which the Neumann system of degree $N$ was retrieved from the Schrödinger-KdV system [5], the Jacobi system of geodesic flow from the Harry-Dym equation [6], and some others [7-9]. Recently, it was found that two typical peakon equations, namely the Camassa-Holm (CH) and Degasperis-Procesi (DP) equations, are connected with Neumann type systems on a symplectic submanifold $[\mathbf{8}, \mathbf{9}]$; and the algebrogeometric solution of $\mathrm{CH}$ equation was obtained in view of a Neumann type system [8]. The finite dimensional integrable Hamiltonian systems (FDIHSs) have been used to derive algebro-geometric solutions for integrable nonlinear evolution equations (INLEEs) in $1+1$ and $2+1$ dimensions [10-15]. Compared with the usage of FDIHS, the application of Neumann type system is a bit more difficult because it adopts a geometry condition confining itself onto the submanifold $[\mathbf{8 , 4 3}]$. It is noted that the Neumann type system can be given a Hamiltonian representation under the Dirac-Poisson bracket and the symmetry constraint (1.2) cuts out a finite dimensional invariant subspace from the infinite dimensional phase space $[\mathbf{1 6}, \mathbf{1 7}]$. This means that the Neumann type system can be used to solve more INLEEs of physical interest.

It is well-known that the ideal aim for the study of INLEEs is to obtain their explicit solutions invoking all kinds of available tools. There are two classes of explicit solutions of integrable systems, i.e., the decreasing and the (quasi) periodic solutions, which are the most interesting from a physical point of view. The quasi periodic solutions expressed by Riemann theta functions can be deduced via the finite-gap integration theory developed in a series of influential works $[\mathbf{1 6 - 2 2}]$ and some monographs [23-26]. Based on the fruitful application of FDIHSs [10-15], the plan of this paper resides in constructing some explicit solutions in the light of Riemann theta functions for INLEEs in both $2+1$ and $1+1$ dimensions through Neumann type systems on the symplectic submanifold, instead of the FDIHSs, Baker-Akhiezer functions, canonical meromorphic functions and axiomatizations of algebro-geometric datum. We take the $2+1$ dimensional CDGKS equation [27]

$$
\begin{aligned}
& 144 v_{t}=-v_{x x x x x}+18 v v_{x x x}+36 v_{x} v_{x x}-432 v^{2} v_{x} \\
& +20 v_{x x y}-312 v v_{y}-72 v_{x} \partial^{-1} v_{y}+80 \partial^{-1} v_{y y}
\end{aligned}
$$


which results from the mKdV hierarchy defined by the INLEE (i.e., the mKdV equation $[\mathbf{2 8}])$,

$$
u_{y}=\frac{1}{4} u_{x x x}-\frac{3}{2} u^{2} u_{x}
$$

as the examples to illustrate the designed scheme. The subscripts $x, y$ and $t$ appended to $u$ and $v$ denote the partial derivatives; $\partial^{-1}$ represents an inverse operator of $\partial=\partial / \partial x$ with the condition $\partial \partial^{-1}=\partial^{-1} \partial=1$; and similar notations will be used frequently later in this paper.

To do this, the $2+1$ dimensional CDGKS equation and the mKdV hierarchy are put into the zero-curvature form in the framework of Lax compatibility [29], where the Lax triad of $2+1$ dimensional CDGKS equation and the Lax pairs of the $\mathrm{mKdV}$ hierarchy are fixed by a sequence of Lenard gradients. Resorting to the nonlinearization of Lax pair $[\mathbf{5}, \mathbf{3 0}, \mathbf{3 1}]$, the $2+1$ dimensional CDGKS, the $\mathrm{mKdV}$ and the fifth-order mKdV equations are decomposed into three FDISs of Neumann type realizing their separation of variables $[\mathbf{3 2}, \mathbf{3 3}]$. It is noted that the DiracPoisson bracket is the directional derivative over the symplectic submanifold. This directly leads to the canonical Hamiltonian equations of Neumann type systems. From two Casimir functions, we deduce the Lax-Moser matrix of Neumann type systems that gives the integrals of motion, elliptic variables, trace formulae and a hyperelliptic curve of KdV type. We also figure out a Lax equation contributing to the involutivity of integrals of motion on the symplectic submanifold. By a recursive formula, we arrive at all the constrained Hamiltonians for the higherorder mKdV equations, and further establish the relationship between Neumann type systems and IDISs, where the involutivity solutions of Neumann type systems give rise to the finite parametric solutions of $1+1$ and $2+1$ dimensional INLEEs and the finite-gap potential of Novikov equation. From the Lax equation and a set of quasi Abel-Jacobi variables, we display the Liouville integrability of a family of Neumann type systems, instead of the Moser's constraint method [3], the r-matrix technique $[\mathbf{8}, \mathbf{3 4}, \mathbf{3 5}]$ and the Lie algebraic approach $[\mathbf{3 6}, \mathbf{3 7}]$. By using the Lax equation, we get the general Dubrovin type equations governing the dynamics of elliptic variables that can be transformed to the evolution of Abel-Jacobi variables, from which the Abel-Jacobi solutions to the Neumann type flows are integrated by the direct quadratures. Finally, depending on the Abel map between the AbelJacobi solutions and the divisors of Jacobi variety, we turn to the Jacobi inversion for getting some explicit solutions to the $2+1$ dimensional CDGKS equation and the mKdV hierarchy by the Riemann theorem and the trace formula.

\section{The Lax representations of INLEEs}

After the advent of inverse spectral transformation solving the Cauchy problem of the $\mathrm{KdV}$ equation [38], in $1968 \mathrm{Lax}$ put the $\mathrm{KdV}$ equation into the Lax form and introduced a hierarchy of INLEEs of KdV type [29]. Subsequently, the Lax pair almost became the starting point for all kinds of research of INLEEs. Before describing the main result of this paper, let us first recall the Lax representations of the $2+1$ dimensional CDGKS equation and the mKdV hierarchy in view of a set of Lenard gradients.

Our point of departure is the linear eigenvalue problem of $2 \times 2$ matrix form

$$
\varphi_{x}=U \varphi, \quad U=u \sigma_{1}+\lambda \sigma_{2}+\sigma_{3}, \quad \varphi=\left(\varphi_{1}, \varphi_{2}\right)^{T},
$$


where

$$
\sigma_{1}=\left(\begin{array}{cc}
1 & 0 \\
0 & -1
\end{array}\right), \quad \sigma_{2}=\left(\begin{array}{ll}
0 & 1 \\
0 & 0
\end{array}\right), \quad \sigma_{3}=\left(\begin{array}{cc}
0 & 0 \\
1 & 0
\end{array}\right),
$$

and $\lambda$ is a constant spectral parameter, $u$ is the spectral potential. To proceed, we now turn to the Lenard gradients $\left\{g_{j}\right\}(-1 \leq j \in \mathbb{Z})$

$$
K g_{j-1}=J g_{j}, \quad J g_{-1}=0, \quad j \geq 0,
$$

where

$$
K=\frac{1}{8} \partial^{3}-\frac{1}{2} \partial u \partial^{-1} u \partial, \quad J=\frac{1}{2} \partial .
$$

Choosing the initial seed $g_{-1}=2$, it is clear that the kernel of $J$ is of dimension 1 and

$$
\operatorname{ker} J=\left\{\varrho g_{-1} \mid \forall \varrho \in \mathbb{R}\right\} \text {. }
$$

Up to $\operatorname{ker} J, g_{j}(j \geq 0)$ can be uniquely figured out by the recursive formula $(2.2)$, for example

$$
g_{0}=2 u, \quad g_{1}=\frac{1}{2} u_{x x}-u^{3}, \quad g_{2}=\frac{1}{8} u_{x x x x}-\frac{5}{4} u^{2} u_{x x}-\frac{5}{4} u u_{x}^{2}+\frac{3}{4} u^{5} .
$$

Postulate that the time-dependent $\varphi$ satisfies another linear eigenvalue problem determined by the Lenard gradients

$$
\varphi_{t_{n}}=V^{(n)} \varphi, \quad n \geq 0
$$

with

$$
\begin{aligned}
V^{(n)=} & \sigma(u, \lambda)[g]=\left(\left(\frac{1}{8} \partial^{2}-\frac{1}{2} u \partial^{-1} u \partial\right) \sigma_{1}\right. \\
& \left.-\lambda\left(\frac{1}{4} \partial+\frac{1}{2} \partial^{-1} u \partial\right) \sigma_{2}+\left(\frac{1}{4} \partial-\frac{1}{2} \partial^{-1} u \partial\right) \sigma_{3}\right)[g]
\end{aligned}
$$

where $g=\sum_{j=0}^{n} g_{j-1} \lambda^{n-j}$ and $\sigma: \mathbb{R} \longrightarrow \operatorname{sl}(2, \mathbb{R})$ is a linear operator. Under the isospectral nature $\lambda_{t_{n}}=0$, we arrive at

$$
U_{t_{n}}-V_{x}^{(n)}+\left[U, V^{(n)}\right]=U_{*}\left(u_{t_{n}}-(K-\lambda J) g\right),
$$

where

$$
U_{*}(\xi)=\left.\frac{d}{d \varepsilon}\right|_{\varepsilon=0} U(u+\varepsilon \xi) .
$$

It follows from (2.1) and (2.6) that the zero-curvature equation

$$
U_{t_{n}}-V_{x}^{(n)}+\left[U, V^{(n)}\right]=0
$$

yields the mKdV hierarchy

$$
u_{t_{n}}=J g_{n} \triangleq X_{n}, \quad n \geq 0,
$$

characterized by the mKdV equation (1.4) with $t_{1}=y$, where the symbol $\triangleq$ means that the LHS is designated by the RHS, for short. It is obvious that (2.1) and (2.4) constitute the Lax pair of (2.7) indicating the integrability of mKdV hierarchy in the sense of Lax compatibility. In particular, the mKdV equation in (2.7) admits the Lax pair (2.1) and

$$
\varphi_{y}=V^{(1)} \varphi, \quad V^{(1)}=\left(\begin{array}{cc}
\lambda u+\frac{1}{4} u_{x x}-\frac{1}{2} u^{3} & \lambda^{2}-\frac{1}{2} \lambda\left(u_{x}+u^{2}\right) \\
\lambda+\frac{1}{2}\left(u_{x}-u^{2}\right) & -\lambda u-\frac{1}{4} u_{x x}+\frac{1}{2} u^{3}
\end{array}\right),
$$


while the next one of fifth-order mKdV equation in (2.7) with $t_{2}=t$ reads

$$
u_{t}=\frac{1}{16} u_{x x x x x}-\frac{5}{2} u u_{x} u_{x x}-\frac{5}{8} u^{2} u_{x x x}-\frac{5}{8} u_{x}^{3}+\frac{15}{8} u^{4} u_{x},
$$

invoking the Lax pair (2.1) and

$$
\varphi_{t}=V^{(2)} \varphi, \quad V^{(2)}=\left(\begin{array}{cc}
V_{11}^{(2)} & V_{12}^{(2)} \\
V_{21}^{(2)} & -V_{11}^{(2)}
\end{array}\right)
$$

where

$$
\begin{aligned}
V_{11}^{(2)} & =\lambda^{2} u+\lambda\left(\frac{1}{4} u_{x x}-\frac{1}{2} u^{3}\right)+\frac{1}{16} u_{x x x x}-\frac{5}{8} u^{2} u_{x x}-\frac{5}{8} u u_{x}^{2}+\frac{3}{8} u^{5}, \\
V_{12}^{(2)} & =\lambda^{3}-\frac{1}{2} \lambda^{2}\left(u_{x}+u^{2}\right)+\lambda\left(-\frac{1}{8} u_{x x x}-\frac{1}{4} u u_{x x}+\frac{1}{8} u_{x}^{2}+\frac{3}{4} u^{2} u_{x}+\frac{3}{8} u^{4}\right), \\
V_{21}^{(2)} & =\lambda^{2}+\frac{1}{2} \lambda\left(u_{x}-u^{2}\right)+\frac{1}{8} u_{x x x}-\frac{1}{4} u u_{x x}+\frac{1}{8} u_{x}^{2}-\frac{3}{4} u^{2} u_{x}+\frac{3}{8} u^{4} .
\end{aligned}
$$

Noticing the fact of $V^{(0)}=U$, it is apparent that the flow variable $t_{0}$ is indeed equivalent to the spatial variable $x$. Since the $1+1$ dimensional systems (1.4) and (2.9) belong to the same integrable hierarchy (2.7), this means that the flows determined by (1.4) and (2.9) are consistent with the property of commutability over the infinite dimensional phase space [39].

Proposition 1. (Ref. [27]) Let $u=u(x, y, t)$ be the compatible solution of INLEEs (1.4) and (2.9). Then

$$
v=u^{2}(x, y, t)
$$

satisfies the $2+1$ dimensional CDGKS equation (1.3).

Proof: Resorting to (1.4) and (2.9), a direct but fussy calculation gives

$$
\begin{gathered}
v v_{x x x}=6 u^{2} u_{x} u_{x x}+2 u^{3} u_{x x x} \\
v_{x x x x x}=20 u_{x x} u_{x x x}+10 u_{x} u_{x x x x}+2 u u_{x x x x x} \\
v_{x x y}=\frac{1}{2} u u_{x x x x x}+u_{x} u_{x x x x}+\frac{1}{2} u_{x x} u_{x x x}-18 u u_{x}^{3}-27 u^{2} u_{x} u_{x x}-3 u^{3} u_{x x x} \\
v_{x} \partial^{-1} v_{y}=u^{2} u_{x} u_{x x}-\frac{1}{2} u u_{x}^{3}-\frac{3}{2} u^{5} u_{x}
\end{gathered}
$$

and

$$
\partial^{-1} v_{y y}=\frac{1}{8} u_{x x} u_{x x x}-\frac{9}{2} u^{2} u_{x} u_{x x}+\frac{9}{2} u^{5} u_{x}-\frac{1}{8} u_{x} u_{x x x x}+\frac{1}{8} u u_{x x x x x} .
$$

Simply inserting the equations (2.12)-(2.16) into (2.9), it is known that (2.11) solves the $2+1$ dimensional CDGKS equation (1.3).

By proposition 1 , it is shown that the $2+1$ dimensional CDGKS equation (1.3) is decomposed into two consistent $1+1$ dimensional integrable systems (1.4) and (2.9), whose compatible solutions give rise to solutions of (1.3) by the transformation (2.11); and an important consequence is that (2.1), (2.8) and (2.10) can be identified as the Lax triad of $2+1$ dimensional CDGKS equation (1.3) delivering its integrability under the Lax compatibility [29].

From the above discussion, the Lax pairs of the $\mathrm{mKdV}$, the fifth-order $\mathrm{mKdV}$ equations and the Lax triad of the $2+1$ dimensional CDGKS equation are specified in our setting, while that of the higher-order mKdV equations are presented 
with the aid of the Lenard gradients. After these preparations, we reduce all the IDISs in question into a family of Neumann type systems through the technique of nonlinearization and present a scheme for getting explicit solutions expressed by Riemann theta functions to the $2+1$ dimensional CDGKS equation and the mKdV hierarchy with the help of Neumann type systems.

\section{The Neumann type systems}

Firstly, only for convenience we make some notations and symbols. Let $p, q$ be a pair of canonical variables on $\mathbb{R}^{2 N}$, where $p=\left(p_{1}, p_{2}, \cdots, p_{N}\right)^{T}$ and $q=$ $\left(q_{1}, q_{2}, \cdots, q_{N}\right)^{T} ;\langle\cdot, \cdot\rangle$ stands for the inner product in $\mathbb{R}^{N} ; \lambda_{1}, \lambda_{2}, \cdots, \lambda_{N}$ are assumed to be $N$ arbitrary given distinct eigenvalues and $\Lambda=\operatorname{diag}\left(\lambda_{1}, \cdots, \lambda_{N}\right)$; $\omega^{2}=d p \wedge d q$ is the symplectic structure on $\mathbb{R}^{2 N}$; and the Poisson bracket of two smooth functions $f=f(p, q)$ and $g=g(p, q)$ is defined by [39]

$$
\{f, g\}=\sum_{j=1}^{N}\left(\frac{\partial f}{\partial q_{j}} \frac{\partial g}{\partial p_{j}}-\frac{\partial f}{\partial p_{j}} \frac{\partial g}{\partial q_{j}}\right)=\left\langle\frac{\partial f}{\partial q}, \frac{\partial g}{\partial p}\right\rangle-\left\langle\frac{\partial f}{\partial p}, \frac{\partial g}{\partial q}\right\rangle .
$$

Stipulate that $p_{j}$ and $q_{j}$ are special solutions of the linear eigenvalue equation (2.1) corresponding to $\lambda_{j}$. Put (2.1) into the form

$$
\left(\begin{array}{c}
p_{j} \\
q_{j}
\end{array}\right)_{x}=\left(\begin{array}{cc}
u & \lambda_{j} \\
1 & -u
\end{array}\right)\left(\begin{array}{c}
p_{j} \\
q_{j}
\end{array}\right), \quad 1 \leq j \leq N .
$$

Noting the rapidly decaying condition of the eigenvalue functions $p_{j}$ and $q_{j}$ at the infinity, a direct calculation leads to

$$
\nabla \lambda_{j}=\delta \lambda_{j} / \delta u=2 p_{j} q_{j}
$$

which satisfies the Lenard eigenvalue equation

$$
\left(K-\lambda_{j} J\right) \nabla \lambda_{j}=0 .
$$

Recalling the Neumann constraint (1.2) in the procedure of nonlinearization $[\mathbf{5}, \mathbf{3 0}$, 31], we get

$$
\langle p, q\rangle=1 .
$$

Differentiating (3.4) with reference to $x$ twice yields

$$
\langle p, p\rangle+\langle\Lambda q, q\rangle=0,
$$

and a Neumann map

$$
u=\frac{\langle\Lambda p, q\rangle}{\langle\Lambda q, q\rangle}=-\frac{\langle\Lambda p, q\rangle}{\langle p, p\rangle}
$$

which connects the spectral potential $u$ with the eigenfunctions $p$ and $q$. Complying with the principle of nonlinearization of Lax pair $[\mathbf{5}, \mathbf{3 0}, \mathbf{3 1}]$, substituting (3.6) back into (3.1), together with (3.4) and (3.5), gives the first Neumann type system of degree $N-1$ in explicit form

$$
\left\{\begin{array}{l}
p_{x}=-\frac{\langle\Lambda p, q\rangle}{\langle p, p\rangle} p+\Lambda q \\
q_{x}=p-\frac{\langle\Lambda p, q\rangle}{\langle\Lambda q, q\rangle} q \\
\langle p, q\rangle=1,\langle p, p\rangle+\langle\Lambda q, q\rangle=0 .
\end{array}\right.
$$

From the mathematical point of view that the independent time variable $t_{k}(k \geq 1)$ is equal to the spatial variable $x$, an analogous operation can also be applied to the 
time-dependent Lax representations (2.8) and (2.10). As a result, one gets another two Neumann type systems in vector form

$$
\left\{\begin{array}{l}
p_{y}=\frac{\langle\Lambda p, q\rangle}{\langle\Lambda q, q\rangle} \Lambda p+\frac{\left\langle\Lambda^{2} p, q\right\rangle}{\langle\Lambda q, q\rangle} p+\frac{\langle\Lambda p, q\rangle}{2\langle\Lambda q, q\rangle^{2}}\left(\langle\Lambda p, p\rangle-\left\langle\Lambda^{2} q, q\right\rangle\right) p-\frac{\langle\Lambda p, q\rangle^{3}}{2\langle\Lambda q, q\rangle^{3}} p \\
+\Lambda^{2} q-\frac{1}{2\langle\Lambda q, q\rangle}\left(\left\langle\Lambda^{2} q, q\right\rangle+\langle\Lambda p, p\rangle\right) \Lambda q-\frac{\langle\Lambda p, q\rangle^{2}}{2\langle\Lambda q, q\rangle^{2}} \Lambda q, \\
q_{y}=\Lambda p+\frac{1}{2\langle\Lambda q, q\rangle}\left(\left\langle\Lambda^{2} q, q\right\rangle+\langle\Lambda p, p\rangle\right) p-\frac{\langle\Lambda p, q\rangle^{2}}{2\langle\Lambda q, q\rangle^{2}} p-\frac{\langle\Lambda p, q\rangle}{\langle\Lambda q, q\rangle} \Lambda q \\
-\frac{\left\langle\Lambda^{2} p, q\right\rangle}{\langle\Lambda q, q\rangle} q-\frac{\langle\Lambda p, q\rangle}{2\langle\Lambda q, q\rangle^{2}}\left(\langle\Lambda p, p\rangle-\left\langle\Lambda^{2} q, q\right\rangle\right) q+\frac{\langle\Lambda p, q\rangle^{3}}{2\langle\Lambda q, q\rangle^{3}} q, \\
\langle p, q\rangle=1, \quad\langle p, p\rangle+\langle\Lambda q, q\rangle=0
\end{array}\right.
$$

and

$$
\left\{\begin{array}{l}
p_{t}=\frac{\langle\Lambda p, q\rangle}{\langle\Lambda q, q\rangle} \Lambda^{2} p+\left(\frac{\left\langle\Lambda^{2} p, q\right\rangle}{\langle\Lambda q, q\rangle}+\frac{\langle\Lambda p, q\rangle}{2\langle\Lambda q, q\rangle^{2}}\left(\langle\Lambda p, p\rangle-\left\langle\Lambda^{2} q, q\right\rangle\right)-\frac{\langle\Lambda p, q\rangle^{3}}{2\langle\Lambda q, q\rangle^{3}}\right) \Lambda p \\
+\frac{\left\langle\Lambda^{3} p, q\right\rangle}{\langle\Lambda q, q\rangle} p+\frac{\langle\Lambda p, q\rangle}{2\langle\Lambda q, q\rangle^{2}}\left(\left\langle\Lambda^{2} p, p\right\rangle-\left\langle\Lambda^{3} q, q\right\rangle\right) p \\
+\frac{\left\langle\Lambda^{2} p, q\right\rangle}{2\langle\Lambda q, q\rangle^{2}}\left(\langle\Lambda p, p\rangle-\left\langle\Lambda^{2} q, q\right\rangle\right) p+\frac{\langle\Lambda p, q\rangle}{2\langle\Lambda q, q\rangle^{3}}\left(\langle\Lambda p, p\rangle^{2}+\left\langle\Lambda^{2} q, q\right\rangle^{2}\right) p \\
-\frac{\langle\Lambda p, q\rangle}{8\langle\Lambda \mu, q\rangle^{3}}\left(\langle\Lambda p, p\rangle+\left\langle\Lambda^{2} q, q\right\rangle\right)^{2} p-\frac{3\langle\Lambda p, q\rangle^{2}\left\langle\Lambda^{2} p, q\right\rangle}{2\langle\Lambda q, q\rangle^{3}} p \\
-\frac{3\langle\Lambda p, q\rangle^{3}}{4\langle\Lambda q, q\rangle^{4}}\left(\langle\Lambda p, p\rangle-\left\langle\Lambda^{2} q, q\right\rangle\right) p+\frac{3\langle\Lambda p, q\rangle^{5}}{8\langle\Lambda q, q)^{5}} p+\Lambda^{3} q-\frac{\langle\Lambda p, q\rangle^{2}}{2\langle\Lambda q, q\rangle^{2}} \Lambda^{2} q \\
-\frac{1}{2\langle\Lambda q, q\rangle}\left(\langle\Lambda p, p\rangle+\left\langle\Lambda^{2} q, q\right\rangle\right) \Lambda^{2} q+\left(\frac{3\left\langle\Lambda^{2} q, q\right\rangle^{2}}{8\langle\Lambda q, q\rangle^{2}}-\frac{\langle\Lambda p, p\rangle^{2}}{8\langle\Lambda q, q\rangle^{2}}\right. \\
-\frac{\left\langle\Lambda^{3} q, q\right\rangle+\left\langle\Lambda^{2} p, p\right\rangle}{2\langle\Lambda q, q\rangle}+\frac{3\langle\Lambda p, q\rangle^{2}\left\langle\Lambda^{2} q, q\right\rangle}{4\langle\Lambda q, q\rangle^{3}}+\frac{\langle\Lambda p, p\rangle\left\langle\Lambda^{2} q, q\right\rangle}{4\langle\Lambda q, q\rangle^{2}} \\
\left.+\frac{3\langle\Lambda p, q\rangle^{4}}{8\langle\Lambda q, q\rangle^{4}}-\frac{\langle\Lambda p, q\rangle\left\langle\Lambda^{2} p, q\right\rangle}{\langle\Lambda q, q\rangle^{2}}-\frac{\langle\Lambda p, q\rangle^{2}\langle\Lambda p, p\rangle}{4\langle\Lambda q, q\rangle^{3}}\right) \Lambda q, \\
q t=\Lambda^{2} p-\frac{\langle\Lambda p, q\rangle^{2}}{2\langle\Lambda q, q\rangle^{2}} \Lambda p+\frac{1}{2\langle\Lambda q, q\rangle}\left(\langle\Lambda p, p\rangle+\left\langle\Lambda^{2} q, q\right\rangle\right) \Lambda p \\
+\left(\frac{\left\langle\Lambda^{3} q, q\right\rangle+\left\langle\Lambda^{2} p, p\right\rangle}{2\langle\Lambda q, q\rangle}-\frac{3\langle\Lambda p, q\rangle^{2}\langle\Lambda p, p\rangle}{4\langle\Lambda q, q\rangle^{3}}+\frac{\langle\Lambda p, q\rangle^{2}\left\langle\Lambda^{2} q, q\right\rangle}{4\langle\Lambda q, q\rangle^{3}}+\frac{3\langle\Lambda p, p\rangle^{2}}{8\langle\Lambda q, q\rangle^{2}}\right. \\
\left.-\frac{\langle\Lambda p, q\rangle\left\langle\Lambda^{2} p, q\right\rangle}{\langle\Lambda q, q\rangle^{2}}-\frac{\left\langle\Lambda^{2} q, q\right\rangle^{2}}{8\langle\Lambda q, q\rangle^{2}}+\frac{\left\langle\Lambda^{2} q, q\right\rangle\langle\Lambda p, p\rangle}{4\langle\Lambda q, q\rangle^{2}}+\frac{3\langle\Lambda p, q\rangle^{4}}{8\langle\Lambda q, q\rangle^{4}}\right) p-\frac{\langle\Lambda p, q\rangle}{\langle\Lambda q, q\rangle} \Lambda^{2} q \\
-\left(\frac{\left\langle\Lambda^{2} p, q\right\rangle}{\langle\Lambda q, q\rangle}+\frac{\langle\Lambda p, q\rangle}{2\langle\Lambda q, q\rangle^{2}}\left(\langle\Lambda p, p\rangle-\left\langle\Lambda^{2} q, q\right\rangle\right)-\frac{\langle\Lambda p, q\rangle^{3}}{2\langle\Lambda q, q\rangle^{3}}\right) \Lambda q-\frac{\left\langle\Lambda^{3} p, q\right\rangle}{\langle\Lambda q, q\rangle} q \\
-\frac{\langle\Lambda p, q\rangle}{2\langle\Lambda q, q\rangle^{2}}\left(\left\langle\Lambda^{2} p, p\right\rangle-\left\langle\Lambda^{3} q, q\right\rangle\right) q-\frac{\left\langle\Lambda^{2} p, q\right\rangle}{2\langle\Lambda q, q\rangle^{2}}\left(\langle\Lambda p, p\rangle-\left\langle\Lambda^{2} q, q\right\rangle\right) q \\
-\frac{\langle\Lambda p, q\rangle}{2\langle\Lambda q, q\rangle^{3}}\left(\langle\Lambda p, p\rangle^{2}+\left\langle\Lambda^{2} q, q\right\rangle^{2}\right) q+\frac{\langle\Lambda p, q\rangle}{8\langle\Lambda q, q\rangle^{3}}\left(\langle\Lambda p, p\rangle+\left\langle\Lambda^{2} q, q\right\rangle\right)^{2} q \\
+\frac{3\langle\Lambda p, q\rangle^{2}\left\langle\Lambda^{2} p, q\right\rangle}{2\langle\Lambda q, q\rangle^{3}} q+\frac{3\langle\Lambda p, q\rangle^{3}}{4\langle\Lambda q, q\rangle^{4}}\left(\langle\Lambda p, p\rangle-\left\langle\Lambda^{2} q, q\right\rangle\right) q-\frac{3\langle\Lambda p, q\rangle^{5}}{8\langle\Lambda q, q\rangle^{5}} q, \\
\langle p, q\rangle=1, \quad\langle p, p\rangle+\langle\Lambda q, q\rangle=0 .
\end{array}\right.
$$

It follows from the Lax representations (2.1), (2.8) and (2.10) that the INLEEs (1.3), (1.4) and (2.9) have been reduced to three finite dimensional nonlinear dynamical systems (3.7), (3.8) and (3.9). At first sight, the systems (3.7), (3.8) and (3.9) contain the same geometry conditions (3.4) and (3.5), similar to that of the classical Neumann system, restricting themselves onto a submanifold of $\mathbb{R}^{2 N}$

$$
M=\left\{(p, q) \in \mathbb{R}^{2 N} \mid F \triangleq\langle p, q\rangle-1=0, G \triangleq\langle p, p\rangle+\langle\Lambda q, q\rangle=0\right\},
$$

where $F$ and $G$ are two Casimir functions; and then the finite dimensional nonlinear dynamical systems (3.7), (3.8) and (3.9) are tentatively called the Neumann type systems. Consequently, the arguments of (3.7), (3.8) and (3.9) should be carried out over $M$ under the symplectic structure $\omega^{2}$ because of the geometry conditions (3.4) and (3.5). Restricting $f$ and $g$ onto $M$, we have

$$
\left\{\left.f\right|_{M},\left.g\right|_{M}\right\}=\{f, g\}+\frac{1}{4\langle p, p\rangle}(\{f, F\}\{G, g\}-\{f, G\}\{F, g\}),
$$


which is nothing else but the Dirac-Poisson bracket $\{f, g\}_{\text {Dirac }}$ equipped with the identities

$$
\left\{p_{k}, p_{l}\right\}_{\text {Dirac }}=0,\left\{p_{k}, q_{l}\right\}_{\text {Dirac }}=p_{k} q_{l}-\delta_{k l},\left\{q_{k}, q_{l}\right\}_{\text {Dirac }}=q_{k} q_{l}-q_{k} p_{l} .
$$

From the definition of Poisson bracket, it is known that the value of $\{f, g\}_{\text {Dirac }}$ is in fact the directional derivative of $f$ in the direction $g$-flow on $M$, which transfers the trouble of geometry conditions to the Dirac-Poisson bracket. Then, the Neumann type systems (3.7), (3.8) and (3.9) can be rewritten as the canonical Hamiltonian equations

$$
\begin{aligned}
& p_{x}=\left\{p, H_{0}\right\}_{\text {Dirac }}, \quad q_{x}=\left\{q, H_{0}\right\}_{\text {Dirac }} \\
& p_{y}=\left\{p, H_{1}\right\}_{\text {Dirac }}, \quad q_{y}=\left\{q, H_{1}\right\}_{\text {Dirac }} \\
& p_{t}=\left\{p, H_{2}\right\}_{\text {Dirac }}, \quad q_{t}=\left\{q, H_{2}\right\}_{\text {Dirac }}
\end{aligned}
$$

with the constrained Hamiltonians

$$
H_{0}=\frac{1}{2}\langle p, p\rangle=-\frac{1}{2}\langle\Lambda q, q\rangle
$$

$$
\begin{aligned}
H_{1}=-\frac{1}{2}\left\langle\Lambda^{2} q, q\right\rangle+ & \frac{1}{2}\langle\Lambda p, p\rangle+\frac{\langle\Lambda p, q\rangle^{2}}{2\langle p, p\rangle}=-\frac{1}{2}\left\langle\Lambda^{2} q, q\right\rangle+\frac{1}{2}\langle\Lambda p, p\rangle-\frac{\langle\Lambda p, q\rangle^{2}}{2\langle\Lambda q, q\rangle}, \\
H_{2}= & -\frac{1}{2}\left\langle\Lambda^{3} q, q\right\rangle+\frac{1}{2}\left\langle\Lambda^{2} p, p\right\rangle-\frac{\langle\Lambda p, q\rangle\left\langle\Lambda^{2} p, q\right\rangle}{\langle\langle q, q\rangle}+\frac{\left\langle\Lambda^{2} q, q\right\rangle^{2}}{4\langle\Lambda q, q\rangle}+ \\
& \frac{\langle\Lambda p, p\rangle\rangle^{2}}{4\langle\Lambda q, q\rangle}+\frac{\langle\Lambda p, q\rangle^{4}}{4\langle\Lambda q, q\rangle^{3}}-\frac{\left\langle\Lambda^{2} q, q\right\rangle\langle\Lambda p, q\rangle^{2}}{2\langle\Lambda q, q\rangle^{2}}+\frac{\langle\Lambda p, p\rangle\langle\Lambda p, q\rangle^{2}}{2\langle\Lambda q, q\rangle^{2}}
\end{aligned}
$$

which indicate that the resulting Neumann type systems (3.7), (3.8) and (3.9) can be viewed as the Hamiltonian flows in the sense of the Dirac-Poisson bracket, whose involutivity is described by $\{\cdot, \cdot\}_{\text {Dirac }}=0$ over the symplectic submanifold $\left(M, \omega^{2}\right)$.

To deal with (3.13), (3.14) and (3.15), it is necessary for us to find their integrals of motion. Let us now introduce a bilinear generating function

$$
G_{\lambda}=\sum_{j=1}^{N} \frac{\nabla \lambda_{j}}{\lambda-\lambda_{j}}=2 Q_{\lambda}(p, q)
$$

which satisfies

$$
(K-\lambda J) G_{\lambda}=0,
$$

where

$$
Q_{\lambda}(\xi, \eta)=\sum_{j=1}^{N} \frac{\xi_{j} \eta_{j}}{\lambda-\lambda_{j}}=\sum_{m=0}^{\infty} \lambda^{-m-1}\left\langle\Lambda^{m} \xi, \eta\right\rangle .
$$

From (2.5), (2.6), (3.19) and (3.20), we work out the desired Lax-Moser matrix

$$
V_{\lambda}=\sigma(u, \lambda)\left[G_{\lambda}\right]=\left(\begin{array}{ll}
Q_{\lambda}(\Lambda p, q) & -\lambda Q_{\lambda}(p, p) \\
Q_{\lambda}(\Lambda q, q) & -Q_{\lambda}(\Lambda p, q)
\end{array}\right),
$$

which satisfies

$$
\left(V_{\lambda}\right)_{x}-\left[U, V_{\lambda}\right]=0
$$


The combination of (3.21) and (3.22) yields the generating function of integrals of motion for the Neumann type system (3.7)

$$
F_{\lambda}=\operatorname{det} V_{\lambda}=\langle p, p\rangle Q_{\lambda}(\Lambda q, q)+Q_{\lambda}(\Lambda p, p) Q_{\lambda}(\Lambda q, q)-Q_{\lambda}^{2}(\Lambda p, q)=\sum_{m=0}^{\infty} F_{m} \lambda^{-m-1}
$$

where

(3.24) $F_{0}=-\langle p, p\rangle^{2}=-\langle\Lambda q, q\rangle^{2}$,

(3.25) $\quad F_{1}=\langle p, p\rangle\left\langle\Lambda^{2} q, q\right\rangle+\langle\Lambda p, p\rangle\langle\Lambda q, q\rangle-\langle\Lambda p, q\rangle^{2}$

(3.26) $F_{2}=\langle p, p\rangle\left\langle\Lambda^{3} q, q\right\rangle+\left\langle\Lambda^{2} p, p\right\rangle\langle\Lambda q, q\rangle+\langle\Lambda p, p\rangle\left\langle\Lambda^{2} q, q\right\rangle-2\langle\Lambda p, q\rangle\left\langle\Lambda^{2} p, q\right\rangle$,

$$
F_{m}=\langle p, p\rangle\left\langle\Lambda^{m+1} q, q\right\rangle+\sum_{j=0}^{m-1}\left|\begin{array}{ll}
\left\langle\Lambda^{j+1} p, p\right\rangle & \left\langle\Lambda^{m-j} p, q\right\rangle \\
\left\langle\Lambda^{j+1} p, q\right\rangle & \left\langle\Lambda^{m-j} q, q\right\rangle
\end{array}\right|, \quad m \geq 3 .
$$

The INLEEs (1.3), (1.4) and (2.9) have been performed the separation of variables on $\left(M, \omega^{2}\right)$ through the Neumann type systems (3.7), (3.8) and (3.9). As for the higher-order $\mathrm{mKdV}$ equation (2.7) $(n \geq 3)$, we introduce a sequence of Neumann type systems $\left(H_{m}, \omega^{2}, M\right)$

$$
p_{t_{m}}=\left\{p, H_{m}\right\}_{\text {Dirac }}, \quad q_{t_{m}}=\left\{q, H_{m}\right\}_{\text {Dirac }}, \quad m \geq 3,
$$

where

$$
H_{m}=-\frac{F_{m}}{4 H_{0}}-\frac{1}{2 H_{0}} \sum_{i+j=m, i, j \geq 1} H_{i} H_{j},
$$

which constitute the integrable decomposition of the higher-order mKdV equations (2.7) $(n \geq 3)$ (see section 5 below). Moreover,

$$
F_{0}+4 H_{0}^{2}=0, \quad F_{1}+4 H_{0} H_{1}=0, \quad F_{2}+2 H_{1}^{2}+4 H_{0} H_{2}=0,
$$

all the constrained Hamiltonians $\left\{H_{m}\right\}$ can be put into a uniform

$$
\lambda F_{\lambda}=-2 H_{\lambda}^{2},
$$

where

$$
H_{\lambda}=\sum_{m=0}^{\infty} H_{m} \lambda^{-m}
$$

is the generating function of $\left\{H_{m}\right\}(m \geq 0)$. Based on the constrained Hamiltonians (3.16)-(3.18) and (3.29), in what follows we specify the Liouville integrability of a sequence of Neumann type systems (3.7), (3.8), (3.9) and (3.28).

\section{The Liouville integrability}

To prove the Liouville integrability of a sequence of Neumann type systems (3.7), (3.8), (3.9) and (3.28), for the present we turn to the generating function method [3]. Let $F_{\lambda}$ be the constrained Hamiltonian on $\left(M, \omega^{2}\right)$, and designate the flow variables of $F_{\lambda}, F_{m}, H_{\lambda}$ and $H_{m}$ by $\tau_{\lambda}, \tau_{m}, t_{\lambda}$ and $t_{m}$, respectively. A direct calculation gives a canonical Hamiltonian equation

$$
\frac{d}{d \tau_{\lambda}}\left(\begin{array}{c}
p_{k} \\
q_{k}
\end{array}\right)=\left(\begin{array}{c}
\left\{p_{k}, F_{\lambda}\right\}_{\text {Dirac }} \\
\left\{q_{k}, F_{\lambda}\right\}_{\text {Dirac }}
\end{array}\right)=W\left(\lambda, \lambda_{k}\right)\left(\begin{array}{c}
p_{k} \\
q_{k}
\end{array}\right),
$$


where

$$
W(\lambda, \mu)=\frac{2 \mu}{\lambda-\mu} V_{\lambda}+2 Q_{\lambda}(\Lambda p, q) \sigma_{1}+2 Q_{\lambda}(\Lambda q, q) \sigma_{3}
$$

THEOREM 1. Let $\lambda$ and $\mu$ be two complex parameters $(\lambda \neq \mu)$. The Lax-Moser matrix $V_{\mu}$ satisfies a Lax equation over the symplectic submanifold $\left(M, \omega^{2}\right)$

$$
\frac{d V_{\mu}}{d \tau_{\lambda}}=\left\{V_{\mu}, F_{\lambda}\right\}_{\text {Dirac }}=\left[W(\lambda, \mu), V_{\mu}\right]
$$

Proof: Only for brevity, set

$$
\mathcal{E}_{k}=\left(\begin{array}{cc}
\lambda_{k} p_{k} q_{k} & -\lambda_{k} p_{k}^{2} \\
\lambda_{k} q_{k}^{2} & -\lambda_{k} p_{k} q_{k}
\end{array}\right)=\lambda_{k}\left(\begin{array}{c}
p_{k} \\
q_{k}
\end{array}\right)\left(p_{k}, q_{k}\right)\left(\begin{array}{cc}
0 & -1 \\
1 & 0
\end{array}\right) .
$$

It is not difficult to calculate from (4.1) that

$$
\frac{d \mathcal{E}_{k}}{d \tau_{\lambda}}=\left[W\left(\lambda, \lambda_{k}\right), \mathcal{E}_{k}\right]
$$

By using (4.1) and (4.4), a direct but lengthy calculation gives

$$
\begin{aligned}
& \frac{d V_{\mu}}{d \tau_{\lambda}}=-\frac{d\langle p, p\rangle}{d \tau_{\lambda}} \sigma_{2}+\sum_{k=1}^{N} \frac{1}{\mu-\lambda_{k}} \frac{d \mathcal{E}_{k}}{d \tau_{\lambda}} \\
& =\sum_{k=1}^{N} \frac{1}{\mu-\lambda_{k}}\left[\frac{2 \lambda_{k}}{\lambda-\lambda_{k}} V_{\lambda}+2 Q_{\lambda}(\Lambda p, q) \sigma_{1}+2 Q_{\lambda}(\Lambda q, q) \sigma_{3}, \mathcal{E}_{k}\right] \\
& =\sum_{k=1}^{N} \frac{1}{\mu-\lambda_{k}} \frac{2 \lambda_{k}}{\lambda-\lambda_{k}}\left[V_{\lambda}, \mathcal{E}_{k}\right]+\left[2 Q_{\lambda}(\Lambda p, q) \sigma_{1}+2 Q_{\lambda}(\Lambda q, q) \sigma_{3}, \sum_{k=1}^{N} \frac{1}{\mu-\lambda_{k}} \mathcal{E}_{k}\right] \\
& =\frac{2}{\lambda-\mu}\left[V_{\lambda}, \mu V_{\mu}-\lambda V_{\lambda}\right]+\left[2 Q_{\lambda}(\Lambda p, q) \sigma_{1}+2 Q_{\lambda}(\Lambda q, q) \sigma_{3}, V_{\mu}\right] \\
& +2\left[-V_{\lambda}+Q_{\lambda}(\Lambda p, q) \sigma_{1}+Q_{\lambda}(\Lambda q, q) \sigma_{3},\langle p, p\rangle \sigma_{2}\right]=\left[W(\lambda, \mu), V_{\mu}\right] .
\end{aligned}
$$

Corollary 1.

$$
\begin{gathered}
\left\{F_{\mu}, F_{\lambda}\right\}_{\text {Dirac }}=0, \quad \lambda, \mu \in \mathbb{C}, \\
\left\{F_{j}, F_{k}\right\}_{\text {Dirac }}=0, \quad j, k=1,2, \cdots .
\end{gathered}
$$

Proof: From the Lax equation (4.3), it is clear that $F_{\mu}=\operatorname{det} V_{\mu}$ is the constant of motion along the $\tau_{\lambda}$-flow; by the Dirac-Poisson bracket, we get

$$
\left\{F_{\mu}, F_{\lambda}\right\}_{\text {Dirac }}=\frac{d F_{\mu}}{d \tau_{\lambda}}=\frac{d}{\tau_{\lambda}} \operatorname{det} V_{\mu}=0 .
$$

Simply substituting (3.23) into (4.5) yields (4.6) by comparing the same powers of $\lambda$ and $\mu$.

COROLlary 2.

$$
\begin{gathered}
\left\{H_{\mu}, H_{\lambda}\right\}_{\text {Dirac }}=0, \quad \forall \lambda, \mu \in \mathbb{C}, \\
\left\{H_{j}, H_{k}\right\}_{\text {Dirac }}=0, \quad j, k=0,1,2, \cdots .
\end{gathered}
$$


Proof: Recalling the Leibniz rule of Dirac-Poisson bracket, it follows from (4.5) that

$$
\left\{H_{\mu}, H_{\lambda}\right\}_{\text {Dirac }}=\frac{\sqrt{\lambda \mu}}{8 \sqrt{F_{\lambda} F_{\mu}}}\left\{F_{\mu}, F_{\lambda}\right\}_{\text {Dirac }}=0,
$$

then inserting (3.32) into (4.7) immediately results in (4.8).

In addition to the involutivity of integrals of motion, the other essential element for Liouville integrability is the functional independence, which means a sufficient number of integrals of motion. In the remainder of this section, we start with the Lax-Moser matrix (3.21) to specify the functional independence of $\left\{F_{m}\right\}$ and $\left\{H_{m}\right\}$ $(m \geq 0)$ by a set of quasi Abel-Jacobi variables. From (3.21) and (4.2), we denote

$$
V_{\lambda}=\left(\begin{array}{cc}
V_{\lambda}^{11} & V_{\lambda}^{12} \\
V_{\lambda}^{21} & -V_{\lambda}^{11}
\end{array}\right), \quad W(\lambda, \mu)=\left(\begin{array}{cc}
W_{\lambda}^{11} & W_{\lambda}^{12} \\
W_{\lambda}^{21} & -W_{\lambda}^{11}
\end{array}\right)
$$

and put $F_{\lambda}$ into the form

$$
F_{\lambda}=-V_{\lambda}^{12} V_{\lambda}^{21}-\left(V_{\lambda}^{11}\right)^{2}=\sum_{j=1}^{N} \frac{E_{j}}{\lambda-\lambda_{j}}
$$

where

$$
E_{j}=\langle p, p\rangle \lambda_{j} q_{j}^{2}+\sum_{k=1, k \neq j}^{N} \frac{\lambda_{k} \lambda_{j}\left(p_{k} q_{j}-p_{j} q_{k}\right)^{2}}{\lambda_{k}-\lambda_{j}} .
$$

It follows from (3.21) and (4.10) that $F_{\lambda}, V_{\lambda}^{12}$ and $V_{\lambda}^{21}$ are the rational polynomial functions of $\lambda$ with the simple pole $\lambda_{j}(j=1,2, \cdots, N)$. Thus, we define

$$
\begin{gathered}
F_{\lambda}=-\langle p, p\rangle^{2} \frac{b(\lambda)}{a(\lambda)}=-\langle p, p\rangle^{2} \frac{R(\lambda)}{a^{2}(\lambda)}=-\langle\Lambda q, q\rangle^{2} \frac{R(\lambda)}{a^{2}(\lambda)} \\
V_{\lambda}^{12}=-\lambda Q_{\lambda}(p, p)=-\langle p, p\rangle \frac{\lambda m(\lambda)}{a(\lambda)} \\
V_{\lambda}^{21}=Q_{\lambda}(\Lambda q, q)=\langle\Lambda q, q\rangle \frac{n(\lambda)}{a(\lambda)}
\end{gathered}
$$

with

$$
\begin{gathered}
a(\lambda)=\prod_{k=1}^{N}\left(\lambda-\lambda_{k}\right), \quad m(\lambda)=\prod_{k=1}^{N-1}\left(\lambda-\mu_{k}\right), \quad n(\lambda)=\prod_{k=1}^{N-1}\left(\lambda-\nu_{k}\right), \\
b(\lambda)=\prod_{k=1}^{N-1}\left(\lambda-\lambda_{N+k}\right), \quad R(\lambda)=a(\lambda) b(\lambda)=\prod_{k=1}^{2 N-1}\left(\lambda-\lambda_{k}\right),
\end{gathered}
$$

where $\mu_{1}, \mu_{2}, \cdots, \mu_{N-1}$ and $\nu_{1}, \nu_{2}, \cdots, \nu_{N-1}$ are two sets of elliptic variables of the Neumann type systems (3.7), (3.8), (3.9) and (3.28).

By the Dirac-Poisson bracket and (3.31), a simple computation leads to

$$
\frac{d F_{\lambda}}{d t_{\lambda}}=\left\{F_{\lambda}, H_{\lambda}\right\}_{\text {Dirac }}=-2 \lambda^{-1} H_{\lambda}\left\{H_{\lambda}, H_{\lambda}\right\}_{\text {Dirac }}=0,
$$

which means that $\left\{F_{m}\right\}(m \geq 0)$ are the conservation integrals for all the Neumann type systems (3.7), (3.8), (3.9) and (3.28), and hence are independent of flow 
variable $t_{m}(m \geq 0)$. Write $\alpha^{2}=\langle p, p\rangle^{2}=\langle\Lambda q, q\rangle^{2}$, for short. Setting $\lambda=\mu_{k}$ and $\lambda=\nu_{k},(4.10)$ in combination with (4.12) gives

$$
\left.V_{\lambda}^{11}\right|_{\lambda=\mu_{k}}=\frac{\alpha \sqrt{R\left(\mu_{k}\right)}}{a\left(\mu_{k}\right)},\left.\quad V_{\lambda}^{11}\right|_{\lambda=\nu_{k}}=\frac{\alpha \sqrt{R\left(\nu_{k}\right)}}{a\left(\nu_{k}\right)}, \quad 1 \leq k \leq N-1 .
$$

By using the Lax equation (4.1) over $\left(M, \omega^{2}\right)$, we readily derive

$$
\left\{\begin{array}{l}
\frac{d V_{\mu}^{12}}{d \tau_{\lambda}}=2\left(W^{11}(\lambda, \mu) V_{\mu}^{12}-W^{12}(\lambda, \mu) V_{\mu}^{11}\right), \\
\frac{d V_{\mu}^{21}}{d \tau_{\lambda}}=2\left(W^{21}(\lambda, \mu) V_{\mu}^{11}-W^{11}(\lambda, \mu) V_{\mu}^{21}\right) .
\end{array}\right.
$$

Appealing to (4.2), (4.13), (4.14), (4.16) and (4.17), we figure out the general Dubrovin type equations

$$
\left\{\begin{array}{l}
\frac{1}{4 \alpha \sqrt{R\left(\mu_{k}\right)}} \frac{d \mu_{k}}{d \tau_{\lambda}}=\frac{\lambda m(\lambda)}{a(\lambda)\left(\lambda-\mu_{k}\right) m^{\prime}\left(\mu_{k}\right)}, \\
\frac{1}{4 \alpha \sqrt{R\left(\nu_{k}\right)}} \frac{d \nu_{k}}{d \tau_{\lambda}}=-\frac{\lambda n(\lambda)}{a(\lambda)\left(\lambda-\nu_{k}\right) n^{\prime}\left(\nu_{k}\right)}, \quad 1 \leq k \leq N-1,
\end{array}\right.
$$

which governs the dynamics of elliptic variables $\mu_{k}$ and $\nu_{k}$. Applying the Lagrange interpolation formula to (4.18), it is not difficult for us to obtain that

$$
\left\{\begin{array}{l}
\sum_{k=1}^{N-1} \frac{\mu_{k}^{N-1-j}}{4 \alpha \sqrt{R\left(\mu_{k}\right)}} \frac{d \mu_{k}}{d \tau_{\lambda}}=\frac{\lambda^{N-j}}{a(\lambda)}, \\
\sum_{k=1}^{N-1} \frac{\nu_{k}^{N-1-j}}{4 \alpha \sqrt{R\left(\nu_{k}\right)}} \frac{d \nu_{k}}{d \tau_{\lambda}}=-\frac{\lambda^{N-j}}{a(\lambda)},
\end{array}\right.
$$

To deal with (4.19), let us consider the Riemann surface $\Gamma$ of hyperelliptic curve of genus $N-1$

$$
\Gamma: \quad \xi^{2}=R(\lambda),
$$

with the holomorphic differentials

$$
\tilde{\omega}_{j}=\frac{\lambda^{N-1-j} d \lambda}{4 \alpha \sqrt{R(\lambda)}}, \quad 1 \leq j \leq N-1,
$$

which form a vector space of dimension $N-1$ in view of the Riemann-Roch theorem [40]. Corresponding to the same $\lambda$, there exist two points on different sheets of $\Gamma$ denoted by

$$
P(\lambda)=(\lambda, \sqrt{R(\lambda)}), \quad P_{-}(\lambda)=(\lambda,-\sqrt{R(\lambda)}) .
$$

Let $P_{0}$ be a fixed point on $\Gamma$. Introduce the quasi Abel-Jacobi variables

$$
\tilde{\phi}_{j}=\sum_{k=1}^{N-1} \int_{P_{0}}^{P\left(\mu_{k}\right)} \tilde{\omega}_{j}, \quad \tilde{\psi}_{j}=\sum_{k=1}^{N-1} \int_{P_{0}}^{P\left(\nu_{k}\right)} \tilde{\omega}_{j}, \quad 1 \leq j \leq N-1 .
$$

It follows from (4.22) that (4.19) can be cast into a format

$$
\frac{d \tilde{\phi}_{j}}{d \tau_{\lambda}}=\frac{\lambda^{N-j}}{a(\lambda)}, \quad \frac{d \tilde{\psi}_{j}}{d \tau_{\lambda}}=-\frac{\lambda^{N-j}}{a(\lambda)}, \quad 1 \leq j \leq N-1
$$

Proposition 2. $\left\{F_{0}, F_{1}, \cdots, F_{N-2}\right\}$ described by (3.24)-(3.27) are functionally independent over the symplectic submanifold $\left(M, \omega^{2}\right)$. 
Proof: According to $[\mathbf{3 9}]$, then we need only prove the linear independence of the differentials $d F_{0}, d F_{1}, \cdots, d F_{N-2}$. According to (3.23), we have

$$
\frac{d \tilde{\phi}_{j}}{d \tau_{\lambda}}=\left\{\tilde{\phi}_{j}, F_{\lambda}\right\}_{\text {Dirac }}=\sum_{k=0}^{\infty}\left\{\tilde{\phi}_{j}, F_{k}\right\}_{\text {Dirac }} \lambda^{-k-1}=\sum_{k=0}^{\infty} \frac{d \tilde{\phi}_{j}}{d \tau_{k}} \lambda^{-k-1} .
$$

Following (4.23), we derive

$$
\frac{d \tilde{\phi}_{j}}{d \tau_{\lambda}}=\frac{\lambda^{N-j}}{a(\lambda)}=\sum_{k=0}^{\infty} A_{k} \lambda^{-k-j}
$$

where

$$
\begin{gathered}
A_{-k}=0(k \geq 1), \quad A_{0}=1, \quad A_{1}=s_{1}, \\
A_{k}=\frac{1}{k}\left(s_{k}+\sum_{i+j=k, i, j \geq 1} s_{i} A_{j}\right), \quad k \geq 2,
\end{gathered}
$$

with $s_{k}=\lambda_{1}^{k}+\cdots+\lambda_{N}^{k}$. Comparing the same power of $\lambda$ on the RHSs of (4.24) and (4.25) gives

$$
\frac{d \tilde{\phi}_{j}}{d \tau_{k}}=A_{k+1-j}
$$

Denoting $\tilde{\phi}=\left(\tilde{\phi}_{1}, \cdots, \tilde{\phi}_{N-1}\right)^{T}$, we achieve

$$
\left(\frac{d \tilde{\phi}}{d \tau_{0}}, \cdots, \frac{d \tilde{\phi}}{d \tau_{N-2}}\right)=\left(\begin{array}{ccccc}
1 & A_{1} & A_{2} & \cdots & A_{N-2} \\
& 1 & A_{1} & \cdots & A_{N-3} \\
& & \ddots & \ddots & \vdots \\
& & & \ddots & A_{1} \\
& 0 & & & 1
\end{array}\right) \triangleq A
$$

It is assumed that there are $N-1$ constants $\gamma_{0}, \gamma_{1}, \cdots, \gamma_{N-2}$ satisfying the identity

$$
\sum_{k=0}^{N-2} \gamma_{k} d F_{k}=0
$$

Conforming to the relation between the Poisson bracket and $\omega^{2}$, we arrive at

$$
0=\sum_{k=0}^{N-2} \gamma_{k} \omega^{2}\left(I d F_{k}, I d \tilde{\phi}_{j}\right)=\sum_{k=0}^{N-2} \gamma_{k}\left\{\tilde{\phi}_{j}, F_{k}\right\}_{\text {Dirac }}=\sum_{k=0}^{N-2} \gamma_{k} \frac{d \tilde{\phi}_{j}}{d \tau_{k}}
$$

which implies the functional independence of $\left\{F_{0}, F_{1}, \cdots, F_{N-2}\right\}$ because $\operatorname{det} A=$ 1.

Note: As for proposition 2, it should be remarked at this point that Ma had already developed another systematical way to handle the functional independence of integrals of motion by using the so-called epsilon technique presented on the basis of polynomials $[\mathbf{4 1}, \mathbf{4 2}]$. Actually, here we can also attain the functional independence of $\left\{F_{0}, F_{1}, \cdots, F_{N-2}\right\}$ given by (3.24)-(3.27) under the epsilon technique and then provide the alternative proof in the Appendix A.

Proposition 3. $\left\{H_{0}, H_{1}, \cdots, H_{N-2}\right\}$ given by (3.16)-(3.18) and (3.29) are functionally independent on the symplectic submanifold $\left(M, \omega^{2}\right)$. 
Proof: Following the recursive formula (3.29), it is easy to see that

$$
\left(\begin{array}{c}
d F_{0} \\
d F_{1} \\
d F_{2} \\
\vdots \\
d F_{N-2}
\end{array}\right)=\left(\begin{array}{cccccc}
-8 H_{0} & 0 & 0 & 0 & \cdots & 0 \\
-4 H_{1} & -4 H_{0} & 0 & 0 & \cdots & 0 \\
\ddots & \ddots & \ddots & \ddots & \vdots & \vdots \\
-4 H_{N-3} & * & * & * & -4 H_{0} & 0 \\
-4 H_{N-2} & * & * & * & * & -4 H_{0}
\end{array}\right)\left(\begin{array}{c}
d H_{0} \\
d H_{1} \\
d H_{2} \\
\vdots \\
d H_{N-2}
\end{array}\right)
$$

which indicates the functional independence of $\left\{H_{0}, H_{1}, \cdots, H_{N-2}\right\}$ on $M$. In a future step, combining corollary 2 with proposition 3 , we come to the Liouville integrability for all the concerned Neumann type systems.

THEOREM 2. The Neumann type systems (3.7), (3.8), (3.9) and (3.28) are completely integrable in the Liouville sense.

Remark: It follows from theorem 2 that Neumann type flows stemmed from the $2+1$ dimensional CDGKS equation and the $\mathrm{mKdV}$ hierarchy commute with each other on $\left(M, \omega^{2}\right)$; that is to say, arbitrary two Neumann type systems $\left(H_{i}, \omega^{2}, M\right)$ and $\left(H_{j}, \omega^{2}, M\right)(i, j=0,1,2, \cdots)$ are consistent, which means that there exists a smooth function in variables of $t_{i}$ and $t_{j}$ giving the involutivity solution of the two Neumann type systems [39].

\section{Relation between Neumann type systems and IDISs}

The FDISs reduced from INLEEs via the nonlinearization of Lax pair $[\mathbf{5}, \mathbf{3 0}, \mathbf{3 1}]$ not only enrich the content of integrable systems, but also pave an effective way to solve INLEEs. An exhaustive treatment of FDIHSs has been conducted for getting algebro-geometric solutions of INLEEs in $1+1$ and $2+1$ dimensions [10-15], where the crucial point is that the relation between INLEEs and FDIHSs is established and the Bargmann map yields the finite-gap potential. Based on this observation, in this section we specify the interrelation between INLEEs and Neumann type systems, where the involutivity solutions of Neumann type systems also give rise to the finite parametric solutions of INLEEs and the finite-gap potential of Novikov equation.

Introduce a generating function of the Lenard gradients $\left\{g_{k}\right\}$

$$
g_{\lambda}=g_{-1}+\sum_{k=0}^{\infty} g_{k} \lambda^{-k-1}
$$

It turns out that $g_{\lambda}$ is a special solution of Lenard eigenvalue equation

$$
(K-\lambda J) g_{\lambda}=0 .
$$

Because the kernel of $J$ is of dimension one with the only basis $g_{-1}$, applying $J^{-1} K$ to the Neumann constraint (1.2) $k$ times, we achieve

$$
\sum_{j=1}^{N} \lambda_{j}^{k} \nabla \lambda_{j}=g_{k-1}+c_{2} g_{k-2}+\cdots+c_{k+1} g_{-1}, \quad k \geq 1,
$$

where $c_{2}, c_{3}, \cdots, c_{k+1}$ are constants of integration. It is not difficult to obtain from (5.3) that

$$
G_{\lambda}=c_{\lambda} g_{\lambda}, \quad c_{\lambda}=\sum_{k=0}^{\infty} c_{k+1} \lambda^{-k-1}, \quad c_{1}=1 .
$$


Due to the rapidly decaying condition of $u$ at infinity, it follows from (3.21) that

$$
F_{\lambda}=\operatorname{det} \sigma(u, \lambda)\left[c_{\lambda} g_{\lambda}\right]=-\frac{1}{2} \lambda c_{\lambda}^{2}
$$

By (3.31) and the Leibniz rule of Dirac-Poisson bracket, we readily derive

$$
\begin{gathered}
H_{\lambda}=\frac{1}{2} \lambda c_{\lambda}, \\
\frac{d}{d t_{\lambda}}=-\frac{1}{2 c_{\lambda}} \frac{d}{d \tau_{\lambda}} .
\end{gathered}
$$

THEOREM 3. Let $\left(p\left(x, t_{n}\right), q\left(x, t_{n}\right)\right)^{T}(n \geq 1)$ be an involutivity solution of $H_{0^{-}}$ and $H_{n}$-flows on $\left(M, \omega^{2}\right)$. Then

$$
u\left(x, t_{n}\right)=\frac{\left\langle\Lambda p\left(x, t_{n}\right), q\left(x, t_{n}\right)\right\rangle}{\left\langle\Lambda q\left(x, t_{n}\right), q\left(x, t_{n}\right)\right\rangle}=-\frac{\left\langle\Lambda p\left(x, t_{n}\right), q\left(x, t_{n}\right)\right\rangle}{\left\langle p\left(x, t_{n}\right), p\left(x, t_{n}\right)\right\rangle},
$$

is the finite parametric solution of the $n$-th $m K d V$ equation (2.7).

Proof: With the aid of (3.7), a direct calculation gives

$$
J G_{\lambda}=Q_{\lambda}(p, p)+Q_{\lambda}(\Lambda q, q),
$$

by (4.1), one gets

$$
\begin{aligned}
\frac{d\langle\Lambda p, q\rangle}{d \tau_{\lambda}} & =\sum_{j=1}^{N}\left(\lambda_{j} q_{j}\left\{p_{j}, F_{\lambda}\right\}_{\text {Dirac }}+\lambda_{j} p_{j}\left\{q_{j}, F_{\lambda}\right\}_{\text {Dirac }}\right) \\
& =-2 \lambda\langle p, p\rangle\left(Q_{\lambda}(p, p)+Q_{\lambda}(\Lambda q, q)\right)
\end{aligned}
$$

from (4.5), (5.4) and (5.7), the combination of (5.9) and (5.10) results in

$$
\frac{d u}{d t_{\lambda}}=-\frac{1}{2 c_{\lambda}} \frac{d u}{d \tau_{\lambda}}=-\frac{1}{2 c_{\lambda}}\left(-2 \lambda J G_{\lambda}\right)=\lambda J g_{\lambda}=\sum_{k=0}^{\infty} J g_{k} \lambda^{-k},
$$

which means that (5.8) solves the $n$-th mKdV equation (2.7).

Proposition 4. Stipulate that $(p(x, y, t), q(x, y, t))^{T}$ is the involutivity solution of the Neumann type systems (3.7), (3.8) and (3.9). Thus

$$
v(x, y, t)=\frac{\langle\Lambda p(x, y, t), q(x, y, t)\rangle^{2}}{\langle\Lambda q(x, y, t), q(x, y, t)\rangle^{2}}=\frac{\langle\Lambda p(x, y, t), q(x, y, t)\rangle^{2}}{\langle p(x, y, t), p(x, y, t)\rangle^{2}}
$$

is the finite parametric solution of the $2+1$ dimensional CDGKS equation (1.3).

Proof: Supposing that $h_{j}^{t_{j}}\left(x=t_{0}\right)$ is the flow operator of the initial value problem of the Neumann type system $\left(H_{j}, \omega^{2}, M\right), j=0,1,2$, i.e. $\left(p\left(t_{j}\right), q\left(t_{j}\right)\right)^{T}=$ $h_{j}^{t_{j}}\left(p_{0}, q_{0}\right)^{T}$, it is easy to see from (4.8) that $h_{i}^{t_{i}}$ and $h_{j}^{t_{j}}$ commute with each other over $M[39]$. As a result, the involutivity solution of the Neumann type systems (3.7), (3.8) and (3.9) can be written as

$$
\left(\begin{array}{c}
p \\
q
\end{array}\right)=h_{0}^{x} h_{1}^{y}\left\{h_{2}^{t}\left(\begin{array}{c}
p_{0} \\
q_{0}
\end{array}\right)\right\}=h_{0}^{x} h_{2}^{t}\left\{h_{1}^{y}\left(\begin{array}{c}
p_{0} \\
q_{0}
\end{array}\right)\right\},
$$

from theorem 3 we conclude that $u(x, y, t)$ is the compatible solution of the mKdV equation (1.4) and the fifth-order mKdV equation (2.9); together with proposition 1 , we know that (5.11) solves the $2+1$ dimensional CDGKS equation (1.3). 
Summing up, it is known from theorem 3 and proposition 4 that the Neumann type systems (3.7), (3.8), (3.9) and (3.28) constitute the reduced FDISs of the $2+1$ dimensional CDGKS equation and the mKdV hierarchy, where the Neumann map (3.6) exactly yields the finite parametric solution of INLEEs (1.4) and (2.7). Interestingly, analogous to the recipe given in [7], this Neumann map also generates the finite-gap potential of the high-order stationary $\mathrm{mKdV}$ (Novikov) equation, which cuts out a finite dimensional invariant subspace from the infinite dimensional phase space for all the INLEEs in question.

Proposition 5. Let $(p(x), q(x))^{T}$ be a solution of the Neumann type system (3.7). Then

$$
u(x)=\frac{\langle\Lambda p(x), q(x)\rangle}{\langle\Lambda q(x), q(x)\rangle}=-\frac{\langle\Lambda p(x), q(x)\rangle}{\langle p(x), p(x)\rangle},
$$

is the finite-gap potential of the $N$-order stationary $m K d V$ equation

$$
X_{N-1}+\bar{c}_{2} X_{N-2}+\cdots+\bar{c}_{N} X_{0}=0,
$$

where $\bar{c}_{j}=\sum_{k=0}^{j-1} a_{k} c_{j-k}(j=2,3, \cdots, N)$ are some constants of integration.

Proof: Let us first introduce an auxiliary polynomial

$$
a(\lambda)=\prod_{j=1}^{N}\left(\lambda-\lambda_{j}\right)=a_{0} \lambda^{N}+a_{1} \lambda^{N-1}+\cdots+a_{N}, \quad a_{0}=1 .
$$

It follows from (5.3) that

$$
\begin{aligned}
0= & \sum_{j=1}^{N} a\left(\lambda_{j}\right) \nabla \lambda_{j}=\sum_{j=1}^{N}\left(\lambda_{j}^{N}+a_{1} \lambda_{j}^{N-1}+\cdots+a_{N}\right) \nabla \lambda_{j} \\
= & \left(g_{N-1}+c_{2} g_{N-2}+\cdots+c_{N+1} g_{-1}\right)+ \\
& \left(a_{1} g_{N-2}+\cdots+a_{1} c_{N} g_{-1}\right)+\cdots \cdots+\left(a_{N} g_{-1}\right) \\
= & g_{N-1}+\bar{c}_{2} g_{N-2}+\cdots+\bar{c}_{N+1} g_{-1},
\end{aligned}
$$

where $\bar{c}_{N+1}=\sum_{k=0}^{j-1} a_{k} c_{N+1-k}$; therefore, applying the Lenard operator $J$ to the above identity (5.14) directly gives rise to (5.13).

As for proposition 5, in fact, Novikov had noted earlier in 1974 that the finitegap solution is the natural analog of the multi-soliton solution provided that it satisfies the higher-order stationary solition equation [16]. Moreover, he pointed out that this class of solutions would be quasi-periodic rather than periodic with respect to $x$, and hence the solution of the inverse spectral problem associated with finitely many spectral gaps should be posed within the class of quasi-periodic functions. As a matter of this fact, we study the evolution of Neumann type flows under the Abel-Jacobi variables in view of the Liouville condition of the Neumann type systems, and further deduce the Riemann theta function representation of the finite-gap potential (5.12).

\section{The application of Neumann type flows}

The striking point in the Liouville-Arnold theory [39] is the straightening out of various flows on the invariant torus, which displays the basic structure of integrability. A further issue is to find explicit solutions to INLEEs invoking all kinds of available tools from different standpoints. Seen in sections $2-5$, the $2+1$ dimensional CDGKS equation and the $\mathrm{mKdV}$ hierarchy are reduced into a family of completely 
integrable Neumann type systems, and the relationship between the INLEEs and the Neumann type systems is definitely established. Also, it is found that the Neumann type flows are the Hamiltonian flows in the sense of Dirac-Poisson bracket on $\left(M, \omega^{2}\right)$, and the genus of a hyperelliptic curve of KdV type coincides with the degree of freedom of Neumann type systems. Based on these facts, the subsequent objective is to linearize the Neumann type flows under the Abel-Jacobi variables, and further to discuss the Jacobi inversion problem on the Riemann surface for getting explicit solutions.

Assume that

$$
a_{1}, a_{2}, \cdots, a_{N-1}, b_{1}, b_{2}, \cdots, b_{N-1},
$$

are the canonical basis of the homology group of cycles on $\Gamma$ with the intersection numbers

$$
a_{i} \circ a_{j}=b_{i} \circ b_{j}=0, \quad a_{i} \circ b_{j}=\delta_{i j}, \quad i, j=1,2, \cdots, N-1 .
$$

Set

$$
\mathscr{A}_{i j}=\int_{a_{j}} \tilde{\omega}_{i}, \quad C=\left(\mathscr{A}_{i j}\right)^{-1}, \quad 1 \leq i, j \leq N-1,
$$

which results in the normalized holomorphic differentials

$$
\omega_{j}=\sum_{l=1}^{N-1} C_{j l} \tilde{\omega}_{l}, \quad 1 \leq j \leq N-1
$$

with the properties

$$
\begin{gathered}
\int_{a_{i}} \omega_{j}=\sum_{l=1}^{N-1} C_{j l} \int_{a_{i}} \tilde{\omega}_{l}=\sum_{l=1}^{N-1} C_{j l} \mathscr{A}_{l i}=\delta_{j i} \\
B_{i j}=\int_{b_{j}} \omega_{i}, \quad 1 \leq i, j \leq N-1 .
\end{gathered}
$$

Then we have a unit matrix $\delta=\left(\delta_{i j}\right)_{N-1 \times N-1}$ and a symmetric matrix $B=$ $\left(B_{i j}\right)_{N-1 \times N-1}$ with positive-definite imaginary part, where $B$ is used to construct the Riemann theta function $\theta(\zeta, B)$ of $\Gamma[\mathbf{4 0}, \mathbf{4 4}]$

$$
\begin{aligned}
\theta(\zeta) & =\sum_{z \in \mathbb{Z}^{N-1}} \exp (\pi \sqrt{-1}\langle B z, z\rangle+2 \pi \sqrt{-1}\langle\zeta, z\rangle), \quad \zeta \in \mathbb{C}^{N-1}, \\
\langle B z, z\rangle & =\sum_{i, j=1}^{N-1} B_{i j} z_{i} z_{j}, \quad\langle\zeta, z\rangle=\sum_{i=1}^{N-1} z_{i} \zeta_{i} .
\end{aligned}
$$

Let $\delta_{1}, \delta_{2}, \cdots, \delta_{N-1}$ be the column vectors of $\delta$, and $B_{1}, B_{2}, \cdots, B_{N-1}$ be the column vectors of $B$. A known fact is that $\left\{\delta_{k}, B_{k}\right\}(1 \leq k \leq N-1)$ span a lattice $\mathcal{T}$ in $\mathbb{C}^{N-1}$. We hence arrive at the Jacobi variety $J(\overline{\mathcal{T}})$ (that is a quotient space of $\mathbb{C}^{N-1}$ over $\mathcal{T}$ ) and the Abel map $\mathcal{A}$ with a linear extension to the divisor group

$$
\mathcal{A}: \operatorname{Div}(\Gamma) \longrightarrow J(\mathcal{T})
$$

defined by

$$
\mathcal{A}(P)=\int_{P_{0}}^{P} \omega, \quad \mathcal{A}\left(\sum_{k=1}^{N-1} n_{k} P_{k}\right)=\sum_{k=1}^{N-1} n_{k} \mathcal{A}\left(P_{k}\right), \quad \omega=\left(\omega_{1}, \omega_{2}, \cdots, \omega_{N-1}\right)^{T}
$$


which lead to the the Abel-Jacobi variables

$$
\begin{aligned}
& \phi=\mathcal{A}\left(\sum_{k=1}^{N-1} P\left(\mu_{k}\right)\right)=\sum_{k=1}^{N-1} \int_{P_{0}}^{P\left(\mu_{k}\right)} \omega=C \tilde{\phi}, \\
& \psi=\mathcal{A}\left(\sum_{k=1}^{N-1} P\left(\nu_{k}\right)\right)=\sum_{k=1}^{N-1} \int_{P_{0}}^{P\left(\nu_{k}\right)} \omega=C \tilde{\psi} .
\end{aligned}
$$

Let $C_{k}(1 \leq k \leq N-1)$ be the column vector of $C$ defined by (6.1). Designating

$$
S_{k}=\lambda_{1}^{k}+\cdots+\lambda_{2 N-1}^{k}, \quad \tilde{R}\left(\lambda^{-1}\right)=\prod_{j=1}^{2 N-1}\left(1-\lambda_{j} \lambda^{-1}\right),
$$

a direct calculation gives rise to

$$
\frac{1}{\sqrt{\tilde{R}\left(\lambda^{-1}\right)}}=\sum_{k=0}^{\infty} \Lambda_{k} \lambda^{-k}
$$

where

$$
\Lambda_{0}=1, \quad \Lambda_{1}=\frac{1}{2} S_{1}, \quad \Lambda_{k}=\frac{1}{2 k}\left(S_{k}+\sum_{i+j=k, i, j \geq 1} S_{i} \Lambda_{j}\right) .
$$

On the other hand, it follows from (4.12) and (5.5) that

$$
\sqrt{R(\lambda)}=\frac{\sqrt{\lambda}}{\sqrt{2} \alpha} a(\lambda) c_{\lambda} .
$$

THEOREM 4. The Abel-Jacobi variables $\phi$ and $\psi$ straighten out the Neumann type $H_{\lambda}$-flows as

$$
\begin{gathered}
\frac{d \phi}{d t_{\lambda}}=-\sum_{k=0}^{\infty} \Omega_{k} \lambda^{-k}, \quad \frac{d \psi}{d t_{\lambda}}=\sum_{k=0}^{\infty} \Omega_{k} \lambda^{-k}, \\
\frac{d \phi}{d t_{k}}=-\Omega_{k}, \quad \frac{d \psi}{d t_{k}}=\Omega_{k}, \quad k \geq 0,
\end{gathered}
$$

where

$$
\begin{aligned}
& \Omega_{0}=\frac{1}{2 \sqrt{2} \alpha} C_{1}, \quad \Omega_{1}=\frac{1}{2 \sqrt{2} \alpha}\left(\Lambda_{1} C_{1}+C_{2}\right), \\
& \Omega_{k}=\frac{1}{2 \sqrt{2} \alpha}\left(\Lambda_{k} C_{1}+\cdots+\Lambda_{1} C_{k}+C_{k+1}\right), \quad 2 \leq k \leq N-2, \\
& \Omega_{k}=\frac{1}{2 \sqrt{2} \alpha}\left(\Lambda_{k} C_{1}+\cdots+\Lambda_{k-N+2} C_{N-1}\right), \quad k \geq N-1 .
\end{aligned}
$$

Proof: It suffices to prove $(6.9)_{1}$ since the proof of $(6.9)_{2}$ is analogous to that of $(6.9)_{1}$. By $(5.7),(6.5)_{1},(4.23)_{1},(6.8)$ and $(6.6)$, we obtain

$$
\begin{aligned}
& \frac{d \phi}{d t_{\lambda}}=-\frac{1}{2 c_{\lambda}} \frac{d \phi}{d \tau_{\lambda}}=-\frac{1}{2 c_{\lambda}} C \frac{d \tilde{\phi}}{d \tau_{\lambda}} \\
& =-\frac{\lambda^{N}}{2 c_{\lambda} a(\lambda)} \sum_{j=1}^{N-1} C_{j} \lambda^{-j}=-\frac{\lambda}{2 \sqrt{2} \alpha \sqrt{\tilde{R}\left(\lambda^{-1}\right)}} \sum_{j=1}^{N-1} C_{j} \lambda^{-j} \\
& =-\frac{\lambda}{2 \sqrt{2} \alpha} \sum_{k=0}^{\infty} \Lambda_{k} \lambda^{-k} \sum_{j=1}^{N-1} C_{j} \lambda^{-j}=-\sum_{k=0}^{\infty} \Omega_{k} \lambda^{-k}
\end{aligned}
$$


From the Dirac-Poisson bracket (3.11) and (3.32), the comparison of coefficients of $\lambda^{-k}$ on both sides of $(6.9)_{1}$ and $(6.9)_{2}$ gives rise to $(6.10)$.

Based on theorem 4, it is clear that (6.10) can be integrated by direct quadratures

$$
\phi=\phi_{0}-\sum_{k=0}^{\infty} \Omega_{k} t_{k}, \quad \psi=\psi_{0}+\sum_{k=0}^{\infty} \Omega_{k} t_{k},
$$

because $\Omega_{k}$ is a constant of motion determined by $\left\{\lambda_{k}\right\}(1 \leq k \leq 2 N-1)$ and $\left\{F_{m}\right\}(m \geq 0)$. Noticing the commutativity of Neumann type flows over $\left(M, \omega^{2}\right)$, the evolution behavior of various flows becomes very simple

$$
\mathrm{H}_{\mathrm{k}} \text { flow : } \phi=\phi_{0}-\Omega_{k} t_{k}, \quad \psi=\psi_{0}+\Omega_{k} t_{k}, \quad k \geq 0,
$$

and

$$
2+1 \text { dimensional CDGKS flow : }\left\{\begin{array}{l}
\phi=\phi_{0}-\Omega_{0} x-\Omega_{1} y-\Omega_{2} t \\
\psi=\psi_{0}+\Omega_{0} x+\Omega_{1} y+\Omega_{2} t .
\end{array}\right.
$$

From this point on, the remainder of this section is to transform the AbelJacobi solutions (6.14) and (6.15) to the explicit solutions in the spectral potentials $u$ and $v$ by the Jacobi inversion

$$
(\phi, \psi) \longrightarrow\left(\mu_{1}, \mu_{2}, \cdots, \mu_{N-1} ; \nu_{1}, \nu_{2}, \cdots, \nu_{N-1}\right),
$$

which permits abundant diversity in different examples. For this purpose, we use the Riemann theorem [40], for $\phi$ and $\psi$ given by (6.5), which asserts that there exist two constant vectors $M^{(1)}, M^{(2)} \in \mathbb{C}^{N-1}$ (the Riemann constants) such that

- $f^{(1)}(\lambda)=\theta\left(\mathcal{A}(P(\lambda))-\phi-M^{(1)}\right)$ has $N-1$ simple zeros at $\mu_{1}, \cdots, \mu_{N-1}$;

- $f^{(2)}(\lambda)=\theta\left(\mathcal{A}(P(\lambda))-\psi-M^{(2)}\right)$ has $N-1$ simple zeros at $\nu_{1}, \cdots, \nu_{N-1}$.

To make the function $f^{(m)}(\lambda)(m=1,2)$ single value, the Riemann surface $\Gamma$ is cut along by all $a_{k}, b_{k}$ to form a simply connected region, whose boundary is designated by $\gamma$ composed of $4 N-4$ edges

$$
a_{1}^{+} b_{1}^{+} a_{1}^{-} b_{1}^{-} a_{2}^{+} b_{2}^{+} a_{2}^{-} b_{2}^{-} \cdots \cdots a_{N-1}^{+} b_{N-1}^{+} a_{N-1}^{-} b_{N-1}^{-},
$$

where the symbols + and - represent the orientation. Since $\operatorname{deg} R(\lambda)=2 N-1$, $\Gamma$ has only one infinity point. Under the local coordinate $z=\lambda^{-\frac{1}{2}}$, we attain the residue formulae for the power sum of $\left\{\mu_{k}\right\}$ and $\left\{\nu_{k}\right\}$

$$
\begin{aligned}
& \sum_{j=1}^{N-1} \mu_{j}^{k}=I_{k}(\Gamma)-\operatorname{Res}_{z=0} z^{-2 k} d \ln f^{(1)}\left(z^{-2}\right), \\
& \sum_{j=1}^{N-1} \nu_{j}^{k}=I_{k}(\Gamma)-\operatorname{Res}_{z=0} z^{-2 k} d \ln f^{(2)}\left(z^{-2}\right),
\end{aligned}
$$

where

$$
I_{k}(\Gamma)=\frac{1}{2 \pi i} \oint_{\gamma} \lambda^{k} d \ln f^{(m)}(\lambda)=\sum_{j=1}^{N-1} \int_{a_{j}} \lambda^{k} \omega_{j}, \quad m=1,2,
$$

is a constant independent of $\phi$ and $\psi[\mathbf{2 4}]$. 
LEMMA 1. In the local coordinate $z=\lambda^{-\frac{1}{2}}$ near $\infty$, the normalized basis $\omega$ is of the form

$$
\omega=-\sqrt{2} \sum_{k=0}^{\infty} \Omega_{k} z^{2 k} d z
$$

Proof: From (6.2), (4.21), (6.6) and (6.11), a direct calculation yields

$$
\begin{aligned}
\omega & =C \tilde{\omega}=\left(C_{1}, \cdots, C_{N-1}\right)\left(\tilde{\omega}_{1}, \cdots, \tilde{\omega}_{N-1}\right)^{T}=\sum_{j=1}^{N-1} C_{j} \lambda^{-j} \frac{\lambda^{-\frac{1}{2}}}{4 \alpha \sqrt{\tilde{R}\left(\lambda^{-1}\right)}} d \lambda \\
& =\sum_{j=1}^{N-1} C_{j} \lambda^{-j} \sum_{k=0}^{\infty} \Lambda_{k} \lambda^{-k} \frac{1}{4 \alpha \sqrt{\lambda}} d \lambda=-\sqrt{2} \sum_{k=0}^{\infty} \Omega_{k} z^{2 k} d z .
\end{aligned}
$$

It is interesting to find from (6.17) that the asymptotic expansion coefficients of normalized holomorphic differential $\omega$ at the infinity point of $\Gamma$ are nothing else, but the evolution velocities of Abel-Jacobi variables $\phi$ and $\psi$ (up to an absolute constant). Besides, for $P\left(z^{-2}\right)$ in the neighborhood of $\infty$, by lemma 1 we have the asymptotic expansion of $\mathcal{A}(P(\lambda))$

$$
\mathcal{A}(P(\lambda))=-\chi-\sqrt{2} \sum_{k=0}^{\infty} \frac{1}{2 k+1} \Omega_{k} z^{2 k+1}
$$

where $\chi=\int_{\infty}^{P_{0}} \omega$ is a constant of integration. Let $\zeta_{j}$ be the $j$ th component of Riemann theta function $\theta$. Denote $\partial_{j}=\frac{\partial}{\partial \zeta_{j}}$ and $\partial_{j k}=\frac{\partial^{2}}{\partial \zeta_{j} \partial \zeta_{k}}$, etc. Following (6.18), a direct calculation leads to the Maclaurin expansion of $f^{(1)}(\lambda)$ in the local coordinate $z$

$$
\begin{aligned}
& \left.f^{(1)}(\lambda)\right|_{\lambda=\infty}=\left.f^{(1)}\left(z^{-2}\right)\right|_{z=0}=\theta\left(\int_{P_{0}}^{P} \omega-\phi-M^{(1)}\right) \\
& =\theta\left(\int_{\infty}^{P} \omega-\chi-\phi-M^{(1)}\right)=\theta\left(-\sqrt{2} \sum_{k=0}^{\infty} \frac{1}{2 k+1} \Omega_{k} z^{2 k+1}-\chi-\phi-M^{(1)}\right) \\
& =\theta\left(\cdots, \sqrt{2} \sum_{k=0}^{\infty} \frac{1}{2 k+1} \Omega_{k j} z^{2 k+1}+\chi_{j}+\phi_{j}+M_{j}^{(1)}, \cdots\right) \\
& =\theta\left(\cdots, \sqrt{2} \Omega_{0 j} z+\frac{\sqrt{2}}{3} \Omega_{1 j} z^{3}+\frac{\sqrt{2}}{5} \Omega_{2 j} z^{5}+o\left(z^{5}\right)+\chi_{j}+\phi_{j}+M_{j}^{(1)}, \cdots\right) \\
& =\theta_{1}+\left(\sqrt{2} \Omega_{0 j} \partial_{j} \theta_{1}\right) z+\left(\Omega_{0 j} \Omega_{0 k} \partial_{j k} \theta_{1}\right) z^{2}+\frac{\sqrt{2}}{3}\left(\Omega_{1 j} \partial_{j} \theta_{1}+\Omega_{0 j} \Omega_{0 k} \Omega_{0 l} \partial_{j k l} \theta_{1}\right) z^{3} \\
& +\frac{1}{6}\left(4 \Omega_{0 j} \Omega_{1 k} \partial_{j k} \theta_{1}+\Omega_{0 j} \Omega_{0 k} \Omega_{0 l} \Omega_{0 m} \partial_{j k l m} \theta_{1}\right) z^{4}+o\left(z^{4}\right) \\
& =\theta_{1}-\sqrt{2} \theta_{1 x} z+\theta_{1 x x} z^{2}-\frac{\sqrt{2}}{3}\left(\theta_{1 x x x}+\theta_{1 y}\right) z^{3}+\frac{1}{6}\left(\theta_{1 x x x x}+4 \theta_{1 x y}\right) z^{4}+o\left(z^{4}\right),
\end{aligned}
$$

and the formula

$\frac{d \ln f^{(1)}(\lambda)}{d z}=-\sqrt{2} \frac{\theta_{1 x}}{\theta_{1}}+2\left(\ln \theta_{1}\right)_{x x} z-\sqrt{2}\left(\frac{2 \theta_{1 x}^{3}}{\theta_{1}^{3}}-\frac{3 \theta_{1 x} \theta_{1 x x}}{\theta_{1}^{2}}+\frac{\theta_{1 y}+\theta_{1 x x x}}{\theta_{1}}\right) z^{2}+\cdots$, 
where $\theta_{1}=\theta\left(\phi+\chi+M^{(1)}\right)$. Consequently, we derive

$$
\sum_{j=1}^{N-1} \mu_{j}=I_{1}(\Gamma)-2 \partial^{2} \ln \theta_{1}
$$

Continuing an analogous calculation as above, we also have

$$
\sum_{j=1}^{N-1} \nu_{j}=I_{1}(\Gamma)-2 \partial^{2} \ln \theta_{2}
$$

where $\theta_{2}=\theta\left(\psi+\chi+M^{(2)}\right)$. On the other hand, comparing the coefficients of $\lambda^{N-2}$ on both sides of (4.13) and (4.14) gives

$$
\frac{\langle\Lambda p, p\rangle}{\langle p, p\rangle}=\sum_{j=1}^{N} \lambda_{j}-\sum_{j=1}^{N-1} \mu_{j}, \quad \frac{\left\langle\Lambda^{2} q, q\right\rangle}{\langle\Lambda q, q\rangle}=\sum_{j=1}^{N} \lambda_{j}-\sum_{j=1}^{N-1} \nu_{j} .
$$

And further, we have from (3.1) and (3.6) that

$$
u_{x}=\frac{\left\langle\Lambda^{2} q, q\right\rangle}{\langle\Lambda q, q\rangle}-\frac{\langle\Lambda p, p\rangle}{\langle p, p\rangle}
$$

which together with (6.21) yields the trace formula

$$
u_{x}=\sum_{j=1}^{N-1} \mu_{j}-\sum_{j=1}^{N-1} \nu_{j}
$$

Finally, relying on $(6.14),(6.15),(6.19),(6.20)$ and $(6.23)$, we achieve the explicit solution expressed by Riemann theta functions for the $k$-th $(k \geq 1) \mathrm{mKdV}$ equation (2.7)

$$
u\left(x, t_{k}\right)=2 \partial \ln \frac{\theta\left(\Omega_{0} x+\Omega_{k} t_{k}+\psi_{0}+\chi+M^{(2)}\right)}{\theta\left(-\Omega_{0} x-\Omega_{k} t_{k}+\phi_{0}+\chi+M^{(1)}\right)}+\beta_{1},
$$

and a new algebro-geometric solution for the $2+1$ dimensional CDGKS equation $(1.3)$

$$
v(x, y, t)=\left(2 \partial \ln \frac{\theta\left(\Omega_{0} x+\Omega_{1} y+\Omega_{2} t+\psi_{0}+\chi+M^{(2)}\right)}{\theta\left(-\Omega_{0} x-\Omega_{1} y-\Omega_{2} t+\phi_{0}+\chi+M^{(1)}\right)}+\beta_{2}\right)^{2},
$$

where $\beta_{1}$ and $\beta_{2}$ are the integration constants along with the spatial variable $x$.

\section{Conclusions and Discussions}

In the present paper, from the nonlinearization of Lax pair we perform the Neumann type integrable reduction for both $2+1$ and $1+1$ dimensional INLEEs on a symplectic submanifold, and then establish the relationship between the Neumann type systems and the INLEEs that simplifies the procedure of getting explicit solutions. As a result, a class of FDISs of Neumann type (3.7), (3.8), (3.9) and (3.28) are presented, which enrich the content of integrable systems. By a hyperelliptic curve of KdV type, we discuss the evolution behavior of Neumann type flows on the Jacobian of a Riemann surface, from which some explicit solutions of INLEEs in both $2+1$ and $1+1$ dimensions are derived through the Jacobi inversion. As for the concrete process, the Lax matrix is generalized to the Lax-Moser matrix in view of a bilinear generating function and the geometry conditions of Neumann type 
systems. Noting that the Dirac-Poisson bracket is indeed the directional derivative on the symplectic submanifold, a Lax equation over the symplectic submanifold is given to display the involutivity of constrained Hamiltonian, and the functional independence of constrained Hamiltonians is accomplished by virtue of a set of quasi Abel-Jacobi variables (or by the so-called epsilon technique $[\mathbf{4 1}, \mathbf{4 2}]$, see the Appendix A below). Therefore, an improved way is presented to verify the Liouville integrability of a sequence of Neumann (type) systems.

It is evident that the recipe of Neumann type systems is much more complicated than that of FDIHSs, where the essential difficulty is the geometry condition restricting finite dimensional nonlinear dynamical systems onto a submanifold. Nevertheless, the application of Neumann type systems has its own advantage. For instance, the FDIHSs have been adapted to obtain algebro-geometric solutions for quite a few INLEEs, but it does not always work for all, like the derivative NLS [45] and the 2-component Dym equations [46], where the reason is that the spectral potentials can not be expressed as the symmetry functions associated with elliptic variables. However, the Neumann type integrable reduction may provide a passage attempting to construct some explicit solutions for those INLEEs. Apart from this situation, it is also found that the peakon equations appeared in the negative order hierarchy of eigenvalue problems, such as the $\mathrm{CH}$ and DP equations, are closely connected with Neumann type systems $[\mathbf{8}, \mathbf{9}]$. Therefore, it is natural to consider that the Neumann type systems are taken as the bridge in the calculation of explicit solutions of peakon equations. To summarize, the application of Neumann type systems can be regarded as a possible way for getting explicit solutions of INLEEs in both $2+1$ and $1+1$ dimensions.

\section{Appendix A. An alternative proof of the proposition 2}

To establish the functional independence of $\left\{F_{k}\right\}, 0 \leq k \leq N-2$, let us first specify the functional independence of $\left\{E_{j}\right\}, 1 \leq j \leq N$. From [39], we just need to verify the linear independence of differentials $d E_{1}, d E_{2}, \cdots, d E_{N}$. Under the condition of $\epsilon^{2}=1 / N$ and $N+\sum_{j=1}^{N} \lambda_{j}=0$, let $\varrho_{0}=\left(p_{1}, \cdots, p_{N}, q_{1}, \cdots, q_{N}\right)$ be a point of $\left(M, \omega^{2}\right)$ satisfying

$$
p_{i}=\epsilon, \quad q_{i}=\epsilon, \quad \epsilon \neq 0, \quad 1 \leq i \leq N,
$$

where $\epsilon$ is a small parameter. Recalling (4.11), after a straightforward computation we have

$$
\left|\begin{array}{cccc}
\frac{\partial E_{1}}{\partial q_{1}} & \frac{\partial E_{2}}{\partial q_{1}} & \ldots & \frac{\partial E_{N}}{\partial q_{1}} \\
\frac{\partial E_{1}}{\partial q_{2}} & \frac{\partial E_{2}}{\partial q_{2}} & \ldots & \frac{\partial E_{N}}{\partial q_{2}} \\
\cdots & \cdots & \ldots & \cdots \\
\frac{\partial E_{1}}{\partial q_{N}} & \frac{\partial E_{2}}{\partial q_{N}} & \cdots & \frac{\partial E_{N}}{\partial q_{N}}
\end{array}\right|_{\varrho_{0}}=\left(2 N \epsilon^{3}\right)^{N} \prod_{i=1}^{N} \lambda_{i}
$$

which implies that when $\lambda_{i} \neq 0(1 \leq i \leq N)$ the polynomial functions $\left\{E_{j}\right\}$, $1 \leq j \leq N$, are functionally independent over a dense open subset of $\left(M, \omega^{2}\right)$ $[4 \mathbf{4 1}, \mathbf{4 2}]$. It is assumed that there are $N-1$ constants $b_{1}, b_{2}, \cdots, b_{N-1}$, which 
satisfy

$$
\sum_{k=1}^{N-1} b_{k} d F_{k-1}=0 .
$$

Combining (3.23) with (4.10), we arrive at

$$
\sum_{j=1}^{N-1} b_{k} \lambda_{j}^{k-1}=0, \quad 1 \leq j \leq N,
$$

in view of the linear independence of $\left\{d E_{j}\right\}(1 \leq j \leq N)$. Taking out the first $N-1$ equations in (A.4), it is easy to see that

$$
\left|\begin{array}{cccc}
1 & \lambda_{1} & \cdots & \lambda_{1}^{N-2} \\
1 & \lambda_{2} & \cdots & \lambda_{2}^{N-2} \\
\cdots & \cdots & \cdots & \cdots \\
1 & \lambda_{N-1} & \cdots & \lambda_{N-1}^{N-2}
\end{array}\right|=\prod_{1 \leq j<i \leq N-1}\left(\lambda_{i}-\lambda_{j}\right) \neq 0
$$

which results in $b_{k}=0(1 \leq k \leq N-1)$ and the functional independence of $\left\{F_{0}, F_{1}, \cdots, F_{N-2}\right\}$ given by (3.24)-(3.27). This completes the proof.

\section{Acknowledgements}

This work was supported by the National Natural Science Foundation of China (No.11001050) and by the Swiss Government Scholarship under the grant reference 2011.0502/China/OP.

\section{References}

[1] Neumann, C.: De problemate quodam mechanica, quod ad primam integralium ultraellipticorum classem revocatur. J. Reine Angew. Math. 56, 46-69 (1859)

[2] McKean, H.P. and Trubowitz, E.: Hill's operator and hyperelliptic function theory in the presence of infinitely many branch points Commun. Pure Appl. Math. 29, 143-226 (1976)

[3] Moser, J.: Geometry of quadrics and spectral theory. In: The Chern Symposium 1979, Springer-Verlag, Berlin, 147-188 (1980).

[4] Knörrer, H.: Geodesics on quadrics and a mechanical problem of C. Neumann. J. Rein. Angew. Math. 334, 69-78 (1982)

[5] Cao, C.W. and Geng, X.G.: Classical integrable systems generated through nonlinearization of eigenvalue problems. Research Reports in Physics, Berlin: Springer-Verlag, 68-78 (1990)

[6] Zhou, R.G.: Dynamical r-matrices for the constrained Harry-Dym flows. Phys. Lett. A 220, 320-330 (1996)

[7] Chen, J.B.: Neumann type integrable reduction for nonlinear evolution equations in $1+1$ and 2+1 dimensions. J. Math. Phys. 50, 123504 (2009)

[8] Qiao, Z.J.: The Camassa-Holm hierarchy, $N$-dimensional integrable systems, and algebrogeometric solution on a symplectic submanifold. Commun. Math. Phys. 239, 309-341 (2003)

[9] Qiao, Z.J.: Integrable hierarchy, $3 \times 3$ constrained systems and parametric solutions. Acta Appl. Math. 83, 199-220 (2004)

[10] Alber, M.S., Camassa, R., Fedorov, Y.N., Holm, D.D. and Marsden, J.E.: The complex geometry of weak piecewise smooth solutions of integrable nonlinear PDE's of shallow water and Dym type. Commun. Math. Phys. 221, 197-227 (2001)

[11] Zhou, R.G.: The finite-band solution of the Jaulent-Miodek equation. J. Math. Phys. 38, 2535-2546 (1997)

[12] Cao, C.W., Wu, Y.T. and Geng, X.G.: Relation between the Kadometsev-Petviashvili equation and the confocal involutive system. J. Math. Phys. 40, 3948-3970 (1999)

[13] Qiao, Z.J.: r-matrix and algebro-geometric solution for the AKNS system. Theor. Math. Phys. 127, 827-834 (2001) 
[14] Geng, X.G., Dai, H.H. and Zhu, J.Y.: Decomposition of the discrete Ablowitz-Ladik hierarchy. Stud. Appl. Math. 118, 281-312 (2007)

[15] Chen, J.B. and Geng, X.G.: Algebro-geometric solution to the modified KadometsevPetviashvili equation. J. Phys. Soc. Jpn. 74, 2217-2222 (2005)

[16] Novikov, S.P.: The periodic problem for the Korteweg-de Vries eqution. Funct. Anal. Appl. 8, 236-246 (1974)

[17] Lax, P.D.: Periodic solutions of the Korteweg-de Vries eqution. Comm. Pure Appl. Math. 28, 141-188 (1975)

[18] Its, A.R. and Matveev, V.B.: Hill operators with finitely many gaps. Funct. Anal. Appl. 9, 65-66 (1975)

[19] Dubrovin, B.A.: Periodic problem for the Korteweg-de Vries eqution in the class of finite-band potentials. Funct. Anal. Appl. 9, 215-223 (1975)

[20] McKean, H.P. and van Moerbeke, P.: The spectrum of Hill's equation. Invent. Math. 30, $217-274(1975)$

[21] Flaschka, H. and McLaughlin, D.W.: Canonically conjugate variables for the Korteweg-de Vries eqution and the Toda lattice with periodic boundary conditions. Progr. Theoret. Phys. 55, 438-456 (1976)

[22] Krichever, I.M.: An algebraicCgeometrical construction of the Zakharov-Shabat equations and their periodic solutions. Sov. Math. Dokl. 15, 394-397 (1976)

[23] Novikov, S.P, Manakov, S.V, Pitaevskii, L.P. and Zakharov, V.E.: Theory of solitons, the inverse scattering methods. New York: Consultants Bureau, (1984)

[24] Dickey, L.A.: Soliton equations and Hamiltonian systems. Singapore: World Scientific, (1991)

[25] Belokolos, E.D., Bobenko, A.I., Enol'skii, V.Z., Its, A.R. and Matveev, V.B.: AlgebroGeometric Approach to Nonlinear Evolution Equations. Berlin: Springer-Verlag, (1994)

[26] Gesztesy, F. and Holden, H.: Soliton equations and their algebro-geometric solutions. Cambridge: Cambridge University Press, (2003)

[27] Cheng, Y. and Li, Y.S.: Constrints of the $2+1$ dimensional integrable soliton systems J. Phys. A: Math. Gen. 25, 419-431 (1992)

[28] Miura, R.M.: Korteweg-de Vries equation and generalizations. I. A remarkable explicit nonlinear transformation. J. Math. Phys. 9, 1202-1204 (1968)

[29] Lax, P.D.: Integrals of nonlinear equation of evolution and solitary waves. Comm. Pure Appl. Math. 21, 467-490 (1968)

[30] Ma, W.X. and Strampp, W.: An explicit symmetry constraint for the Lax pairs and the adjoint Lax pairs of AKNS systems. Phys. Lett. A 185, 277-286 (1994)

[31] Ma, W.X.: Symmetry constraint of MKdV equations by binary nonlinearization. Physica A, 219, 467-481 (1995)

[32] Zeng, Y.B. and Ma, W.X.: Separation of variables for soliton equations via their binary constrained flows. J. Math. Phys. 40, 6526-6557 (1999)

[33] Ma, W.X. and Zeng, Y.B.: Binary constrained flows and separation of variables for soliton equations. ANZIAM J. 44, 129-139 (2002)

[34] Babelon, O. and Villet C.-M.: Hamiltonian structures and Lax equations. Phys. Lett. B 237, 411-416 (1990)

[35] Zhou, R.G.: Lax representation, r-matrix method, and separation of variables for the Neumann-type restricted flow. J. Math. Phys. 39, 2848-2858 (1998)

[36] Ratiu, T.: The C.Neumann problem as a completely integrable system on an adjoint orbit. Trans. Amer. Math. Soc. 264, 321-329 (1981)

[37] Ratiu, T.: Involution theorems. Lectures notes in Math., Springer-verlag, Berlin and New York, 755, 219-257 (1980)

[38] Gardner, C.S., Greene, J.M., Kruskal, M.D. and Miura, R.M.: Method for solving the KortewegCde Vries equation. Phys. Rev. Lett. 19, 1095-1097 (1967)

[39] Arnold, V.I.: Mathematical methods of classical mechanics. Berlin: Springer-Verlag, (1978)

[40] Griffiths, P. and Harris, J.: Principles of algebraic geometry. New York: Wiley, (1994)

[41] Ma, W.X. and Zhou, Z.X.: Binary symmetry constraints of N-wave interaction equations in $1+1$ and $2+1$ dimensions. J. Math. Phys. 42, 4345-4382 (2001)

[42] Li, Y.S. and Ma, W.X.: A nonconfocal involutive system and constrained flows associated with the MKdV equation. J. Math. Phys. 43, 4950-4962 (2002)

[43] Chen, J.B.: The application of Neumann type systems for solving integrable nonlinear evolution equations. Stud. Appl. Math. 127, 153-190 (2011) 
[44] Mumford, D.: Tata lectures on theta II. Boston: Birkhauser, (1984)

[45] Cao, C.W. and Yang, X.: A (2+1)-dimensional derivative Toda equation in the context of the Kaup-Newell spectral problem. J. Phys. A: Math. Theor. 41, 025203 (2008)

[46] Alber, M.S., Luther, G.G. and Marsden, J.E.: Energy dependent Schrödinger operators and complex Hamiltonian systems on Riemann surfaces. Nonlinearity, 10, 223-241 (1997)

Department of Mathematics, Southeast University, Nanjing, Jiangsu 210096, P.R. China. Section de Mathématiques, Ecole Polytechnique Fédérale de Lausanne, CH-1015 Lausanne, SWitzerland

E-mail address: cjb@seu.edu.cn 